\title{
NEW APPROXIMATION ALGORITHMS FOR A CLASS OF PARTIAL DIFFERENTIAL EQUATIONS DISPLAYING BOUNDARY LAYER BEHAVIOR*
}

\author{
WENFANG CHENG ${ }^{\dagger}$, ROGER TEMAM ${ }^{\ddagger}$, AND XIAOMING WANG§
}

\begin{abstract}
The aim of this article is to propose and study a class of new algorithms which qualitatively and quantitatively capture the behavior of the exact solutions of a class of evolution partial differential equations which display boundary layer behavior. The idea of the new schemes is to incorporate the boundary layer into the Galerkin base in the finite element approximation. Our error estimates demonstrate that the new schemes are effective in the under-resolved region of the classical schemes. Our numerical experiments support the numerical analysis. The design and analysis of the new schemes depend on the detailed analysis of the boundary layer. The development and proof of the asymptotic expansion of the solutions of the partial differential equations are attached as an Appendix.
\end{abstract}

1. Introduction. Many physical problems involve diffusive process with small diffusive coefficients. The Navier-Stokes equations for incompressible Newtonian fluids is such an example where the Newtonian viscosity is usually very small. This kind of small diffusive coefficient coupled with Dirichlet boundary condition (like the no-slip boundary condition in fluid problems) usually produce a thin sharp transition layer near the boundary, called boundary layer. The problem with boundary layer can be illustrated via the following example of Friedrichs (1941): consider the boundary value problem

$$
\begin{aligned}
-\varepsilon u_{x x}^{\varepsilon}-\beta u_{x}^{\varepsilon} & =1, \text { in }(0, L), \\
u^{\varepsilon}(0)=u^{\varepsilon}(L) & =0 .
\end{aligned}
$$

where $\beta>0$ is a fixed parameter. The solution can be calculated explicitly as

$$
u^{\varepsilon}(x)=-\frac{x}{\beta}+\frac{1-e^{-\beta x / \varepsilon}}{1-e^{-\beta L / \varepsilon}} \frac{L}{\beta} .
$$

The corresponding inviscid problem, equipped with up-wind boundary condition is

$$
\begin{aligned}
-\beta u_{x} & =1, \text { in }(0, L), \\
u(L) & =0 .
\end{aligned}
$$

We observe that there is a transition layer located near $x=0$ of thickness $\varepsilon / \beta$ where $u^{\varepsilon}$ changes value from 0 to 1 .

This is somewhat a generic situation even for similar time evolution problems as we shall recall in the Appendix. In numerical approximation of such problems it is then required to resolve such small scales. In fact for the classical methods such as standard finite elements it is well known that they produce oscillations in the underresolved region, i.e., when the mesh size is larger than the boundary layer thickness

\footnotetext{
${ }^{*}$ Received January 5, 2000.

†Institute for Scientific Computing \& Applied Mathematics, Indiana University, Bloomington, IN 47405-5701 (wcheng@cs.indiana.edu).

$\ddagger$ Institute for Scientific Computing \& Applied Mathematics, Indiana University, Bloomington, IN 47405-5701 and Laboratoire d'Analyse Numérique, Université, Paris Sud, 91405 Orsay, France (temam@indiana.edu).

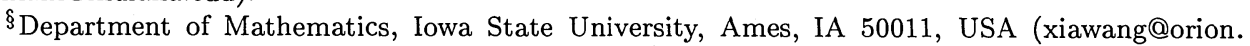
math.iastate.edu).
} 
(see attached figures). On the other hand it is very costly in computational fluid dynamics (CFD) problems if we resolve the boundary layer using a sufficenitly fine mesh and standard methods. Here we would like to propose, study and partially test a new scheme which incorporates the boundary layer into the Galerkin base. We study the scheme on a model problem of a convection-diffusion equation of the following form

$$
\begin{aligned}
\frac{\partial}{\partial t} u^{\varepsilon}-\varepsilon u_{x x}^{\varepsilon}-\beta u_{x}^{\varepsilon} & =f(x, t), \\
u^{\varepsilon}(0, t)=u^{\varepsilon}(L, t) & =0 \\
u^{\varepsilon}(x, 0) & =u_{0}(x),
\end{aligned}
$$

where $\varepsilon$ is a small diffusive coefficient. This model problem is an embedded system of the Navier-Stokes system with non-characteristic boundary conditions:

$$
\begin{aligned}
\frac{\partial}{\partial t} \vec{v}-\varepsilon \Delta \vec{v}+(\vec{v} \cdot \nabla) \vec{v}+\nabla p & =\vec{f}, \\
\nabla \cdot \vec{v} & =0, \\
\left.\vec{v}\right|_{x=0, L} & =(-\beta, 0,0) .
\end{aligned}
$$

Indeed consider a special type of body force $\vec{f}=(0, f(x, t), 0)$ and a special type of solution $\vec{v}=(-\beta, u(x, t), 0)$. We have

$$
\begin{aligned}
\frac{\partial}{\partial x} p & =0 \\
\frac{\partial}{\partial t} u-\varepsilon u_{x x}-\beta u_{x}+\frac{\partial}{\partial y} p & =f \\
\frac{\partial}{\partial z} p & =0 \\
\left.u\right|_{x=0, L} & =0 .
\end{aligned}
$$

Since the equations involve $x, t$ only, we must have

$$
\frac{\partial}{\partial y} p=0
$$

and hence we end up with our one dimensional model problem.

The steady state case of this model problem was studied in Cheng and Temam (1999) first. The idea of our new algorithm is in the same spirit as in that work: namely we try to incorporate the boundary layer into our Galerkin base. In this way we have resolved the boundary layer which enables our scheme to function well even in the region which is under-resolved in the classical case. Our numerical analysis and computations indicate that our method captures qualitatively and quantitatively the behavior of the exact solution even in the under-resolved case (mesh size $>>\varepsilon$ ). A similar idea of incorporating special feature of the equation into the Galerkin base so that the new scheme works in the under-resolved regime of classical scheme can be found in the work of Hou, Wu and Cai (1999) among others. In the steady state case, it is also related to the so called exponential fitting method (see for instance Ascher, Mattheij and Russell, 1988).

The boundary layer behavior of this model evolution problem bears some resemblance to the time independent (steady state) problem studied by Cheng (1999) and 
Cheng and Temam (1999). In particular we show in the Appendix that the boundary layer is located at the outlet (downwind) boundary $(x=0)$ only and is of uniform thickness $\varepsilon / \beta$. This is the same as for the Navier-Stokes equations as was studied by Temam and Wang (1999). The time independent feature of the boundary layer is somewhat a surprise since we generally anticipate the variation of the boundary layer as time evolves. Mathematically this is due to the non-characteristic nature of the boundary (for the inviscid problem) and the incompressibility in the case of Navier-Stokes equations. There is a distinction however between the stationary case and the time evolution case: for the stationary case the boundary layer is of the form $e^{-\beta x / \varepsilon}$ while for the time evolution case secondary boundary layer such as $x e^{-\beta x / \varepsilon}$ appears. The appearance of the secondary boundary layer is due to the resonance effect (see the Appendix for more details). The appearance of this secondary boundary layer also provides us with the choice of whether to incorporate this secondary boundary layer into our Galerkin base. Depending on whether we take into account this secondary boundary layer, we propose two different new schemes. The difference in the numerical results are minimal since the secondary boundary layer is small in the $H^{1}$ norm.

The article is organized as follows. In Section 2 we present the new schemes and in Section 3 we derive error estimates for the new schemes. These error estimates indicate the applicability of the new schemes in the under-resolved region for the classical methods. In Section 4 we present some numerical evidence supporting our numerical analysis. In the Appendix we derive and prove the systematic asymptotic expansion for our model problem for small diffusive coefficient $\varepsilon$.

Application of our new schemes to more realistic models such as the Navier-Stokes system and the comparison of our method to other non-standard methods (such as adaptive, streamline diffusion, etc) will be the subject of future work.

2. The new algorithms. According to the systematic asymptotic expansion developed and proved in the Appendix we know that there exist smooth functions $u^{0}(x, t), u^{1}(x, t)$ and $c_{0}(t), \tilde{c}_{0}(t)$ and a generic constant $\kappa$ independent of $\varepsilon$ such that

$$
\begin{aligned}
& \left\|u^{\varepsilon}-u^{0}-\varepsilon u^{1}-c_{0} e^{-\beta x / \varepsilon}-\tilde{c}_{0} x e^{-\beta x / \varepsilon}\right\|_{L^{\infty}\left(0, T ; H^{2}\right)} \leq \kappa, \\
& \left\|u^{\varepsilon}-u^{0}-\varepsilon u^{1}-c_{0} e^{-\beta x / \varepsilon}-\tilde{c}_{0} x e^{-\beta x / \varepsilon}\right\|_{L^{\infty}\left(0, T ; H^{1}\right)} \leq \kappa \varepsilon .
\end{aligned}
$$

This implies that

$$
\left\|u^{\varepsilon}-c_{0} e^{-\beta x / \varepsilon}-\tilde{c}_{0} x e^{-\beta x / \varepsilon}\right\|_{L^{\infty}\left(0, T ; H^{2}\right)} \leq \kappa,
$$

and

$$
\begin{aligned}
& \left\|u^{\varepsilon}-u^{0}-\varepsilon u^{1}-c_{0} e^{-\beta x / \varepsilon}\right\|_{L^{\infty}\left(0, T ; H^{2}\right)} \leq \kappa \varepsilon^{-\frac{1}{2}} \\
& \left\|u^{\varepsilon}-u^{0}-\varepsilon u^{1}-c_{0} e^{-\beta x / \varepsilon}\right\|_{L^{\infty}\left(0, T ; H^{1}\right)} \leq \kappa \varepsilon^{\frac{1}{2}}
\end{aligned}
$$

Interpolation inequalities imply

$$
\left\|u^{\varepsilon}-u^{0}-\varepsilon u^{1}-c_{0} e^{-\beta x / \varepsilon}\right\|_{L^{\infty}\left(0, T ; H^{\frac{3}{2}}\right)} \leq \kappa,
$$

which further implies

$$
\left\|u^{\varepsilon}-c_{0} e^{-\beta x / \varepsilon}\right\|_{L^{\infty}\left(0, T ; H^{\frac{3}{2}}\right)} \leq \kappa .
$$


These estimates suggest that we have a choice for our new Galerkin basis (classical finite element basis plus boundary layer base).

For fixed $N$, we consider the classical piecewise linear finite element base functions $\varphi_{j}$ of the following form: for $h=\frac{L}{N}$ and for $j=1, \cdots, N-1, x_{j}=j \star h$ and

$$
\begin{gathered}
\varphi_{j}=\left\{\begin{array}{cc}
\frac{1}{h}\left(x-x_{j-1}\right) & \text { for } x_{j-1} \leq x \leq x_{j}, \\
\frac{1}{h}\left(x_{j+1}-x\right) & \text { for } x_{j} \leq x \leq x_{j+1}, \\
0 & \text { elsewhere }
\end{array}\right. \\
\varphi_{0}(x)=e^{-\beta x / \varepsilon}+\left(1-e^{-\frac{\beta L}{\varepsilon}}\right) x / L-1, \\
\tilde{\varphi}_{0}(x)=x e^{-\beta x / \varepsilon}-x e^{-\frac{\beta L}{\varepsilon}}
\end{gathered}
$$

Let

$$
\begin{aligned}
V_{N}=\operatorname{span} & \left\{\varphi_{1}, \cdots, \varphi_{N-1}\right\} \\
\tilde{V}_{N}=\operatorname{span} & \left\{\varphi_{0}, \varphi_{1}, \cdots, \varphi_{N-1}\right\} \\
\tilde{\tilde{V}}_{N}=\operatorname{span} & \left\{\varphi_{0}, \tilde{\varphi}_{0}, \varphi_{1}, \cdots, \varphi_{N-1}\right\} .
\end{aligned}
$$

Our first algorithm takes into consideration the primary boundary layer type base $\varphi_{0}$ only: we consider the problem of finding $\tilde{u}_{N}(t) \in \tilde{V}_{N}(t)$ such that

$$
\begin{aligned}
\left(\frac{\partial \tilde{u}_{N}}{\partial t}, v\right)+\varepsilon\left(\tilde{u}_{N x}, v_{x}\right)-\left(\tilde{u}_{N x}, v\right) & =(f, v), \quad \forall v \in \tilde{V}_{N} \\
\left(\left.\tilde{u}_{N}\right|_{t=0}, v\right) & =\left(u_{0}, v\right), \quad \forall v \in \tilde{V}_{N} .
\end{aligned}
$$

In (2.14) we may replace $\tilde{V}_{N}$ by $V_{N}$ since the initial data $u_{0}$ has no boundary layer by our assumption ( assuming all compatibility conditions are satisfied, see the Appendix for more details) .

Our second algorithm takes into consideration both the primary boundary layer base $\varphi_{0}$ and the secondary boundary layer base $\tilde{\varphi}_{0}$. Hence we seek an approximate solution $\tilde{\tilde{u}}_{N} \in \tilde{\tilde{V}}_{N}$ such that

$$
\begin{aligned}
\left(\frac{\partial \tilde{\tilde{u}}_{N}}{\partial t}, v\right)+\varepsilon\left(\tilde{\tilde{u}}_{N x}, v_{x}\right)-\left(\tilde{\tilde{u}}_{N x}, v\right) & =(f, v), \quad \forall v \in \tilde{\tilde{V}}_{N} \\
\left(\left.\tilde{\tilde{u}}_{N}\right|_{t=0}, v\right) & =\left(u_{0}, v\right), \quad \forall v \in \tilde{\tilde{V}}_{N} .
\end{aligned}
$$

3. Error estimates. In this section, we derive error estimates for the new algorithms proposed in the last section. These error estimates indicate that our scheme should produce satisfactory results when $h<<\varepsilon^{\frac{1}{3}}$. Notice this is well in the underresolved region of the classical methods.

First we notice that thanks to the boundary layer asymptotic expansions (2.6) and (2.3) we have, when combined with classical interpolation results for finite element spaces, that there exist $c_{1}, \cdots, c_{N-1}$ and a constant $\kappa$ independent of $\varepsilon$ such that

$$
\begin{aligned}
\left\|u^{\varepsilon}-\sum_{k=0}^{N-1} c_{k} \varphi_{k}\right\|_{L^{\infty}\left(0, T ; L^{2}\right)} & \leq \kappa h^{\frac{3}{2}}, \\
\left\|u^{\varepsilon}-\sum_{k=0}^{N-1} c_{k} \varphi_{k}\right\|_{L^{\infty}\left(0, T ; H^{1}\right)} & \leq \kappa h^{\frac{1}{2}},
\end{aligned}
$$




$$
\begin{aligned}
& \left\|u^{\varepsilon}-\tilde{c}_{0} \tilde{\varphi}_{0}-\sum_{k=0}^{N-1} c_{k} \varphi_{k}\right\|_{L^{\infty}\left(0, T ; L^{2}\right)} \leq \kappa h^{2} \\
& \left\|u^{\varepsilon}-\tilde{c}_{0} \tilde{\varphi}_{0}-\sum_{k=0}^{N-1} c_{k} \varphi_{k}\right\|_{L^{\infty}\left(0, T ; H^{1}\right)} \leq \kappa h .
\end{aligned}
$$

Of course the c's may be different in each cases, although we use the same notation in all cases.

We then have the following theorem

THEOREM 1. There exists a constant $\kappa=\kappa\left(T, u_{0}, f\right)$ independent of $\varepsilon$ or $h$, such that

$$
\left\|u^{\varepsilon}-\tilde{u}_{N}\right\|_{L^{\infty}\left(0, T ; L^{2}\right)} \leq \kappa\left(T, u_{0}, f\right)\left(\varepsilon^{\frac{1}{2}} h^{\frac{1}{2}}+\frac{h^{\frac{3}{2}}}{\varepsilon^{\frac{1}{2}}}\right)\left(1+\log \frac{T}{h^{2}}\right) .
$$

Proof. Let

$$
\hat{u}_{N}=\sum_{k=0}^{N-1} c_{k} \varphi_{k}
$$

be the interpolation of $u^{\varepsilon}$ in $\tilde{V}_{N}$ such that the interpolation inequalities (3.1) and (3.2) hold with $\hat{u}_{N}$ as the interpolant.

Denote

$$
e_{N}(t)=\tilde{u}_{N}(t)-\hat{u}_{N}(t) .
$$

It is easy to see that we only need to prove the estimate for this error between the approximate solution and the interpolation since

$$
\begin{aligned}
\left\|u^{\varepsilon}-\tilde{u}_{N}\right\|_{L^{\infty}\left(0, T ; L^{2}\right)} & \leq\left\|u^{\varepsilon}-\hat{u}_{N}\right\|_{L^{\infty}\left(0, T ; L^{2}\right)}+\left\|\tilde{u}_{N}-\hat{u}_{N}\right\|_{L^{\infty}\left(0, T ; L^{2}\right)} \\
& \leq \kappa h^{\frac{3}{2}}+\left\|\tilde{u}_{N}-\hat{u}_{N}\right\|_{L^{\infty}\left(0, T ; L^{2}\right)},
\end{aligned}
$$

thanks to the interpolation inequality (3.1).

In order to estimate $e_{N}$ we consider the auxiliary adjoint problem

$$
\begin{aligned}
-\left(\frac{\partial}{\partial s} v_{N}(s), v\right)+a_{\varepsilon}\left(v, v_{N}(s)\right) & =0, \forall v \in V_{N}, s \in(0, t) \\
\left(v_{N}(t)-e_{N}(t), v\right) & =0, \forall v \in V_{N}
\end{aligned}
$$

where

$$
a_{\varepsilon}(u, v)=\varepsilon\left(u_{x}, v_{x}\right)+\beta\left(u, v_{x}\right) .
$$

Denoting

$$
\Theta_{N}(s)=u^{\varepsilon}(s)-\hat{u}_{N}(s),
$$

we see that

$$
\begin{aligned}
\Theta_{N}-e_{N}=u^{\varepsilon}-\hat{u}_{N}-\left(\tilde{u}_{N}-\hat{u}_{N}\right) & =u^{\varepsilon}-\tilde{u}_{N} \\
\left(\Theta_{N}(0)-e_{N}(0), v\right)=\left(u_{0}-\tilde{u}_{N}(0), v\right) & =0, \forall v \in \tilde{V}_{N}, \\
\left(\frac{\partial}{\partial s}\left(\Theta_{N}-e_{N}\right), v\right)+a_{\varepsilon}\left(\Theta_{N}-e_{N}, v\right) & =0, \forall v \in \tilde{V}_{N} .
\end{aligned}
$$


Thus we deduce that

$$
\begin{aligned}
(3.14)\left\|e_{N}(t)\right\|_{L^{2}}^{2}= & \int_{0}^{t}\left[\left(-\frac{\partial}{\partial s} v_{N}(s), e_{N}(s)\right)+a_{\varepsilon}\left(e_{N}(s), v_{N}(s)\right)\right]+\left(v_{N}(t), e_{N}(t)\right) \\
= & \int_{0}^{t}\left[\left(\frac{\partial}{\partial s} e_{N}(s), v_{N}(s)\right)+a_{\varepsilon}\left(e_{N}(s), v_{N}(s)\right)\right]+\left(v_{N}(0), e_{N}(0)\right) \\
= & \int_{0}^{t}\left[\left(\frac{\partial}{\partial s} \Theta_{N}(s), v_{N}(s)\right)+a_{\varepsilon}\left(\Theta_{N}(s), v_{N}(s)\right)\right]+\left(\Theta_{N}(0), v_{N}(0)\right) \\
= & -\int_{0}^{t}\left(\Theta_{N}(s), \frac{\partial}{\partial s} v_{N}(s)\right)+\int_{0}^{t} a_{\varepsilon}\left(\Theta_{N}(s), v_{N}(s)\right)+\left(\Theta_{N}(t), v_{N}(t)\right) \\
\leq & \left\|\Theta_{N}(s)\right\|_{L^{\infty}\left(0, t ; L^{2}\right)}\left\|\frac{\partial}{\partial s} v_{N}(s)\right\|_{L^{1}\left(0, t ; L^{2}\right)} \\
& +\varepsilon\left\|\Theta_{N}\right\|_{L^{\infty}\left(0, t ; H^{1}\right)}\left\|v_{N}\right\|_{L^{1}\left(0, t ; H^{1}\right)}+\beta\left\|\Theta_{N}\right\|_{L^{\infty}\left(0, t ; L^{2}\right)}\left\|v_{N}\right\|_{L^{1}\left(0, t ; H^{1}\right)} \\
& +\left\|\Theta_{N}(t)\right\|_{L^{2}}\left\|e_{N}(t)\right\|_{L^{2}} .
\end{aligned}
$$

Thus it is necessary to estimate various norms of $v_{N}$. We observe that after reversing the time the equation for $v_{N}$ is equivalent to an ODE system

$$
\begin{aligned}
\dot{\eta}+\varepsilon A \eta+\beta B \eta & =0, \\
\eta(0) & =\eta_{0},
\end{aligned}
$$

where $A$ corresponds to the discrete Laplacian and $B$ corresponds to the discretization of $-\frac{\partial}{\partial x}$.

Multiplying equation (3.15) by $\eta$ we deduce

$$
\frac{1}{2} \frac{d}{d s}|\eta(s)|^{2}+\varepsilon\left|A^{\frac{1}{2}} \eta(s)\right|^{2}=0
$$

where we used the skew symmetry of the operator $B$. Hence we have

$$
\begin{aligned}
\left\|v_{N}\right\|_{L^{\infty}\left(0, t ; L^{2}\right)} \leq \kappa\|\eta\|_{L^{\infty}(0, t)} & \leq \kappa\left|\eta_{0}\right| \leq \kappa\left\|e_{N}(t)\right\|_{L^{2}} \\
\left\|v_{N}\right\|_{L^{2}\left(0, t ; H^{1}\right)} \leq \kappa\left\|A^{\frac{1}{2}} \eta\right\|_{L^{2}(0, t)} & \leq \frac{\kappa}{\varepsilon^{\frac{1}{2}}}\left|\eta_{0}\right| \leq \frac{\kappa}{\varepsilon^{\frac{1}{2}}}\left\|e_{N}(t)\right\|_{L^{2}}
\end{aligned}
$$

Multiplying equation (3.15) by $s \dot{\eta}$ we deduce

$$
\begin{aligned}
s|\dot{\eta}(s)|^{2}+\frac{\varepsilon}{2} \frac{d}{d s}\left(s\left|A^{\frac{1}{2}} \eta(s)\right|^{2}\right)-\frac{\varepsilon}{2}\left|A^{\frac{1}{2}} \eta(s)\right|^{2} & \leq \kappa\left|A^{\frac{1}{2}} \eta(s)\right| s|\dot{\eta}(s)| \\
& \leq \frac{1}{2} s|\dot{\eta}(s)|^{2}+\kappa s\left|A^{\frac{1}{2}} \eta(s)\right|^{2} .
\end{aligned}
$$

When combined with the $L^{2}\left(0, t ; A^{\frac{1}{2}}\right)$ estimate, this implies

$$
\begin{aligned}
\left|A^{\frac{1}{2}} \eta(s)\right| & \leq \frac{\kappa}{\varepsilon s^{\frac{1}{2}}}\left|\eta_{0}\right|, \\
\left|s^{\frac{1}{2}} \dot{\eta}(s)\right|_{L^{2}(0, t)} & \leq \frac{\kappa}{\varepsilon^{\frac{1}{2}}}\left|\eta_{0}\right| .
\end{aligned}
$$

Next we take the time derivative of equation (3.15) and find

$$
\ddot{\eta}(s)+\varepsilon A \dot{\eta}(s)+\beta B \dot{\eta}(s)=0 .
$$


Multiplying this equation by $s^{2} \dot{\eta}(s)$ we deduce that

$$
\frac{1}{2} \frac{d}{d s}|s \dot{\eta}(s)|^{2}-s|\dot{\eta}(s)|^{2}+\varepsilon\left|s A^{\frac{1}{2}} \dot{\eta}(s)\right|^{2}=0 .
$$

This implies

$$
|\dot{\eta}(s)| \leq \frac{\kappa}{\varepsilon^{\frac{1}{2}} s}\left|\eta_{0}\right|,
$$

which further implies, together with the inverse Poincaré inequality, that

$$
\begin{aligned}
\int_{0}^{1}|\dot{\eta}(s)| d s & =\int_{0}^{h^{2}}|\dot{\eta}(s)| d s+\int_{h^{2}}^{t}|\dot{\eta}(s)| d s \\
& \leq \int_{0}^{h^{2}}|\varepsilon A \eta(s)+\beta B \eta(s)| d s+\kappa \int_{h^{2}}^{t} \frac{1}{\varepsilon^{\frac{1}{2}} s}\left|\eta_{0}\right| d s \\
& \leq \int_{0}^{h^{2}}\left(\varepsilon \frac{\kappa}{h^{2}}|\eta(s)|+\frac{\kappa}{h}|\eta(s)|\right)+\kappa \varepsilon^{-\frac{1}{2}}\left|\eta_{0}\right| \log \frac{T}{h^{2}} \\
& \leq \kappa\left|\eta_{0}\right|\left(\varepsilon+h+\varepsilon^{-\frac{1}{2}} \log \frac{T}{h^{2}}\right) \\
& \leq \kappa \varepsilon^{-\frac{1}{2}}\left|\eta_{0}\right|\left(1+\log \frac{T}{h^{2}}\right) .
\end{aligned}
$$

Utilizing these relations in the estimate for $\left\|e_{N}\right\|^{2}$ we obtain

$$
\begin{aligned}
\left\|e_{N}(t)\right\|_{L^{2}}^{2} \leq & \kappa \frac{h^{\frac{3}{2}}}{\varepsilon^{\frac{1}{2}}}\left\|e_{N}(t)\right\|_{L^{2}}\left(1+\log \frac{T}{h^{2}}\right)+\kappa h^{\frac{3}{2}}\left\|e_{N}(t)\right\|_{L^{2}} \\
& +\kappa \varepsilon^{\frac{1}{2}} h^{\frac{1}{2}}\left\|e_{N}(t)\right\|_{L^{2}}+\kappa \frac{h^{\frac{3}{2}}}{\varepsilon^{\frac{1}{2}}}\left\|e_{N}(t)\right\|_{L^{2}} \\
\leq & \kappa\left(\varepsilon^{\frac{1}{2}} h^{\frac{1}{2}}+\frac{h^{\frac{3}{2}}}{\varepsilon^{\frac{1}{2}}}\left(1+\log \frac{T}{h^{2}}\right)\right)\left\|e_{N}(t)\right\|_{L^{2}}
\end{aligned}
$$

which implies the result of the theorem.

This completes the proof of this theorem.

If we are willing to assume more regularity on the data $u_{0}, f$ and more compatibility conditions so that the interpolation inequalities (3.1) and (3.2) hold for the time derivative as well (this is possible due to our analysis in the Appendix) we are able to improve the previous error estimate by removing the logarithmic term or deriving a bound on the error which is independent of $\varepsilon$. More precisely we have

THEOREM 2. Let $\tilde{u}_{N}$ be the solution of the boundary layer base truncation equations (2.13) - (2.14). Let $u$ be the exact solution of the linear convection-diffusion equation (1.6) - (1.8). Then there exists a constant $\kappa=\kappa\left(T, u_{0}, f\right)$ independent of $\varepsilon$ or $h$ such that

$$
\left\|u-\tilde{u}_{N}\right\|_{L^{\infty}\left(0, T ; L^{2}\right)} \leq \kappa\left(T, u_{0}, f\right)\left(\varepsilon^{\frac{1}{2}} h^{\frac{1}{2}}+\frac{h^{\frac{3}{2}}}{\varepsilon^{\frac{1}{2}}}\right),
$$

and

$$
\left\|u-\tilde{u}_{N}\right\|_{L^{\infty}\left(0, T ; L^{2}\right)} \leq \kappa\left(T, u_{0}, f\right) h^{\frac{1}{2}}
$$


provided that the data are smooth $\left(C^{6}\right)$ and satisfy the compatibility conditions (to order 4) (see the Appendix).

Proof. Thanks to the systematic asymptotic expansion in the Appendix under added smoothness and compatibility, we see that, there exist $c_{0}^{\prime}(t), \tilde{c}_{0}^{\prime}(t)$ and a generic constant $\kappa$ independent of $\varepsilon$, such that

$$
\left\|\frac{\partial}{\partial t} u^{\varepsilon}-\frac{\partial}{\partial t} u^{0}-\varepsilon \frac{\partial}{\partial t} u^{1}-c_{0}^{\prime} e^{-\beta x / \varepsilon}-\tilde{c}_{0}^{\prime} x e^{-\beta x / \varepsilon}\right\|_{L^{2}\left(0, T ; H^{2}\right)} \leq \kappa \varepsilon^{\frac{1}{2}} .
$$

This implies that

$$
\left\|\frac{\partial}{\partial t} u^{\varepsilon}-c_{0}^{\prime} e^{-\beta x / \varepsilon}\right\|_{L^{2}\left(0, T ; H^{\frac{3}{2}}\right)} \leq \kappa .
$$

By applying the usual interpolation theory to $\frac{\partial}{\partial t} u^{\varepsilon}-c_{0}^{\prime} e^{-\beta x / \varepsilon}$, we see that, there exist $c_{k}^{\prime}$ such that

$$
\begin{aligned}
\left\|\frac{\partial}{\partial t} u^{\varepsilon}-\sum_{k=0}^{N-1} c_{k}^{\prime} \varphi_{k}\right\|_{L^{2}\left(0, T ; L^{2}\right)} & \leq \kappa h^{\frac{3}{2}}, \\
\left\|\frac{\partial}{\partial t} u^{\varepsilon}-\sum_{k=0}^{N-1} c_{k}^{\prime} \varphi_{k}\right\|_{L^{2}\left(0, T ; H^{1}\right)} & \leq \kappa h^{\frac{1}{2}} .
\end{aligned}
$$

Since the initial data $u_{0}$ is smooth, there exist $c_{k}(0), k=1, \cdots, N-1$ and a generic constant $\kappa$ (independent of $\varepsilon$ ) such that

$$
\begin{aligned}
& \left\|u_{0}-\sum_{k=1}^{N-1} c_{k}(0) \varphi_{k}\right\|_{L^{2}} \leq \kappa h^{2}, \\
& \left\|u_{0}-\sum_{k=1}^{N-1} c_{k}(0) \varphi_{k}\right\|_{H^{1}} \leq \kappa h .
\end{aligned}
$$

Now we define

$$
\begin{aligned}
& c_{k}(t)=c_{k}(0)+\int_{0}^{t} c_{k}^{\prime}(s) d s, k=1, \cdots, N-1, \\
& c_{0}(t)=\int_{0}^{t} c_{0}^{\prime}(s) d s
\end{aligned}
$$

and denote

$$
\hat{u}_{N}(x, t)=\sum_{k=0}^{N-1} c_{k}(t) \varphi_{k}(x) \in \tilde{V}_{N} .
$$

We deduce, after integrating (3.31) and (3.32) in time

$$
\begin{aligned}
\left\|u^{\varepsilon}-\hat{u}_{N}\right\|_{L^{\infty}\left(0, T ; L^{2}\right)} & \leq \kappa h^{\frac{3}{2}}, \\
\left\|u^{\varepsilon}-\hat{u}_{N}\right\|_{L^{\infty}\left(0, T ; H^{1}\right)} & \leq \kappa h^{\frac{1}{2}}, \\
\left\|\frac{\partial}{\partial t} u^{\varepsilon}-\frac{\partial}{\partial t} \hat{u}_{N}\right\|_{L^{2}\left(0, T ; L^{2}\right)} & \leq \kappa h^{\frac{3}{2}}, \\
\left\|\frac{\partial}{\partial t} u^{\varepsilon}-\frac{\partial}{\partial t} \hat{u}_{N}\right\|_{L^{2}\left(0, T ; H^{1}\right)} & \leq \kappa h^{\frac{1}{2}} .
\end{aligned}
$$


By setting $v=\tilde{u}_{N}$ in (2.13), we see that

$$
\left\|\tilde{u}_{N}\right\|_{L^{\infty}\left(0, T ; L^{2}\right)} \leq \kappa .
$$

We now consider the difference of $u$ and $\tilde{u}_{N}$; we have

$$
\left(\frac{\partial}{\partial t}\left(u-\tilde{u}_{N}\right), v\right)+\varepsilon\left(u_{x}-\tilde{u}_{N x}, v_{x}\right)-\left(u_{x}-\tilde{u}_{N x}, v\right)=0 \quad \forall v \in \tilde{V}_{N},
$$

(3.44) $\left.\quad \tilde{u}_{N}\right|_{t=0} \in V_{N},\left(\left.\tilde{u}_{N}\right|_{t=0}, v\right)=\left(u_{0}, v\right) \quad \forall v \in V_{N}$.

Equation (3.44) implies

$$
\left\|u_{0}-\left.\tilde{u}_{N}\right|_{t=0}\right\|_{L^{2}} \leq \kappa\left\|u_{0}\right\|_{H^{2}} \cdot h^{2} \leq \kappa h^{2} ;
$$

indeed, (3.44) can be written as

$$
\left(u_{0}-\left.\tilde{u}_{N}\right|_{t=0}, v\right)=0 \quad \forall v \in V_{N},\left.\tilde{u}_{N}\right|_{t=0} \in V_{N} .
$$

Hence, for every $\bar{u} \in V_{N}$,

$$
\begin{aligned}
& \left(u_{0}-\left.\tilde{u}_{N}\right|_{t=0}, u_{0}-\left.\tilde{u}_{N}\right|_{t=0}\right) \\
= & \left(u_{0}-\left.\tilde{u}_{N}\right|_{t=0}, u_{0}-\bar{u}\right)+\left(u_{0}-\left.\tilde{u}_{N}\right|_{t=0}, \bar{u}-\left.\tilde{u}_{N}\right|_{t=0}\right) \\
= & \left(u_{0}-\left.\tilde{u}_{N}\right|_{t=0}, u_{0}-\bar{u}\right) ;
\end{aligned}
$$

we thus have

$$
\left\|u_{0}-\left.\tilde{u}_{N}\right|_{t=0}\right\|_{L^{2}}^{2} \leq\left\|u_{0}-\left.\tilde{u}_{N}\right|_{t=0}\right\|_{L^{2}}\left\|u_{0}-\bar{u}\right\|_{L^{2}}, \quad \forall \bar{u} \in V_{N},
$$

and (3.45) follows from the usual interpolation inequalities.

Now notice that

8) $\frac{1}{2} \frac{d}{d t}\left\|u-\tilde{u}_{N}\right\|_{L^{2}}^{2}+\varepsilon\left\|u_{x}-\tilde{u}_{N x}\right\|_{L^{2}}^{2}$

$=\left(\frac{\partial}{\partial t}\left(u-\tilde{u}_{N}\right), u-\tilde{u}_{N}\right)+\varepsilon\left(u_{x}-\tilde{u}_{N x}, u_{x}-\tilde{u}_{N x}\right)-\left(u_{x}-\tilde{u}_{N x}, u-\tilde{u}_{N}\right)$

$=\left(\frac{\partial}{\partial t}\left(u-\tilde{u}_{N}\right), \hat{u}_{N}-\tilde{u}_{N}\right)+\left(\frac{\partial}{\partial t}\left(u-\tilde{u}_{N}\right), u-\hat{u}_{N}\right)$

$+\varepsilon\left(u_{x}-\tilde{u}_{N x}, \hat{u}_{N x}-\tilde{u}_{N x}\right)+\varepsilon\left(u_{x}-\tilde{u}_{N x}, u_{x}-\hat{u}_{N x}\right)$

$-\left(u_{x}-\tilde{u}_{N x}, \hat{u}_{N}-\tilde{u}_{N}\right)-\left(u_{x}-\tilde{u}_{N x}, u-\hat{u}_{N}\right)$

$=\left(\frac{\partial}{\partial t}\left(u-\tilde{u}_{N}\right), u-\hat{u}_{N}\right)+\varepsilon\left(u_{x}-\tilde{u}_{N x}, u_{x}-\hat{u}_{N x}\right)-\left(u_{x}-\tilde{u}_{N x}, u-\hat{u}_{N}\right)$

$\leq\left(\frac{\partial}{\partial t}\left(u-\tilde{u}_{N}\right), u-\hat{u}_{N}\right)+\varepsilon\left\|u-\tilde{u}_{N}\right\|_{H^{1}}\left\|u-\hat{u}_{N}\right\|_{H^{1}}+\left\|u-\tilde{u}_{N}\right\|_{H^{1}}\left\|u-\hat{u}_{N}\right\|_{L^{2}}$

$\leq\left(\frac{\partial}{\partial t}\left(u-\tilde{u}_{N}\right), u-\hat{u}_{N}\right)+\frac{\varepsilon}{2}\left\|u-\tilde{u}_{N}\right\|_{H^{1}}^{2}+\kappa \varepsilon\left\|u-\hat{u}_{N}\right\|_{H^{1}}^{2}+\kappa \varepsilon^{-1}\left\|u-\hat{u}_{N}\right\|_{L^{2}}^{2}$

$\leq\left(\frac{\partial}{\partial t}\left(u-\tilde{u}_{N}\right), u-\hat{u}_{N}\right)+\frac{\varepsilon}{2}\left\|u-\tilde{u}_{N}\right\|_{H^{1}}^{2}+\kappa\left(\varepsilon h+\frac{h^{3}}{\varepsilon}\right)$

(Thanks to (3.38) and (3.39)).

Hence, we have

$$
\frac{d}{d t}\left\|u-\tilde{u}_{N}\right\|_{L^{2}}^{2}+\varepsilon\left\|u-\tilde{u}_{N}\right\|_{H^{1}}^{2} \leq 2\left(\frac{\partial}{\partial t}\left(u-\tilde{u}_{N}\right), u-\hat{u}_{N}\right)+\kappa\left(\varepsilon h+\frac{h^{3}}{\varepsilon}\right) .
$$


Integrating (3.49) in time and utilizing (3.45), we find that

$$
\begin{aligned}
& \left\|\left(u-\tilde{u}_{N}\right)(t)\right\|_{L^{2}}^{2}+\varepsilon \int_{0}^{t}\left\|\left(u-\tilde{u}_{N}\right)(s)\right\|_{H^{1}}^{2} d s \\
\leq & \kappa\left(h^{4}+\varepsilon h+\frac{h^{3}}{\varepsilon}\right)+2\left(\left(u-\tilde{u}_{N}\right)(t),\left(u-\hat{u}_{N}\right)(t)\right) \\
& \quad-2 \int_{0}^{t}\left(\left(u-\tilde{u}_{N}\right)(s),\left(\frac{\partial u}{\partial t}-\frac{\partial \hat{u}_{N}}{\partial t}\right)(s)\right) d s \\
\leq & \kappa\left(h^{2}+\varepsilon h+\frac{h^{3}}{\varepsilon}\right)+\frac{1}{2}\left\|\left(u-\tilde{u}_{N}\right)(t)\right\|_{L^{2}}^{2}+2\left\|\left(u-\hat{u}_{N}\right)(t)\right\|_{L^{2}}^{2} \\
& +\frac{1}{2} \int_{0}^{t}\left\|\left(u-\tilde{u}_{N}\right)(s)\right\|_{L^{2}}^{2} d s+2 \int_{0}^{t}\left\|\left(\frac{\partial u}{\partial t}-\frac{\partial \hat{u}_{N}}{\partial t}\right)(s)\right\|_{L^{2}}^{2} d s \\
\leq & \kappa\left(h^{4}+\varepsilon h+\frac{h^{3}}{\varepsilon}+h^{3}\right)+\frac{1}{2}\left\|\left(u-\tilde{u}_{N}\right)(t)\right\|_{L^{2}}^{2}+\frac{1}{2} \int_{0}^{t}\left\|\left(u-\tilde{u}_{N}\right)(s)\right\|_{L^{2}}^{2}
\end{aligned}
$$

(Thanks to (3.40)).

This further implies

$$
\begin{gathered}
\left\|\left(u-\tilde{u}_{N}\right)(t)\right\|_{L^{2}}^{2}+2 \varepsilon \int_{0}^{t}\left\|\left(u-\tilde{u}_{N}\right)(s)\right\|_{H^{1}}^{2} d s \\
\leq \kappa\left(h^{4}+\varepsilon h+\frac{h^{3}}{\varepsilon}+h^{3}\right)+\int_{0}^{t}\left\|\left(u-\tilde{u}_{N}\right)(s)\right\|_{L^{2}}^{2} d s .
\end{gathered}
$$

Now we apply the usual Gronwall inequality (see for instance J. Hale, 1980); we find

$$
\left\|u-\tilde{u}_{N}\right\|_{L^{\infty}\left(0, T ; L^{2}\right)} \leq \kappa\left(T, u_{0}, f\right)\left(\varepsilon^{\frac{1}{2}} h^{\frac{1}{2}}+\frac{h^{\frac{3}{2}}}{\varepsilon^{\frac{1}{2}}}\right) .
$$

This indicates that the scheme $(2.13)$ - (2.14) could capture the qualitative behavior in the under-resolved case $(h>>\varepsilon)$, as long as $h^{\frac{3}{2}} \varepsilon^{-\frac{1}{2}}<<1$ or $h<<\varepsilon^{\frac{1}{3}}$, ( e.g. $h<<10^{-1}$ if $\varepsilon=10^{-3}$ ).

An alternative way to derive the convergence rate for the under-resolved scheme is as follows. Notice that

$$
\begin{aligned}
\left|\left(u_{x}-\tilde{u}_{N x}, u-\hat{u}_{N}\right)\right| & =\left|\left(u-\tilde{u}_{N}, u_{x}-\hat{u}_{N x}\right)\right| \\
& \leq\left\|u-\tilde{u}_{N}\right\|_{L^{2}}\left\|u_{x}-\hat{u}_{N x}\right\|_{L^{2}} \\
& \leq \frac{1}{4}\left\|u-\tilde{u}_{N}\right\|_{L^{2}}^{2}+\left\|u_{x}-\hat{u}_{N x}\right\|_{L^{2}}^{2} \\
& \leq \frac{1}{4}\left\|u-\tilde{u}_{N}\right\|_{L^{2}}^{2}+\kappa h
\end{aligned}
$$

(Thanks to $(3.39))$.

Hence we have, instead of (3.48) and (3.49)

$$
\begin{aligned}
& \frac{d}{d t}\left\|u-\tilde{u}_{N}\right\|_{L^{2}}^{2}+\varepsilon\left\|u-\tilde{u}_{N}\right\|_{H^{1}}^{2} \\
\leq & \kappa h+2\left(\frac{\partial}{\partial t}\left(u-\tilde{u}_{N}\right), u-\hat{u}_{N}\right)+\frac{1}{2}\left\|u-\tilde{u}_{N}\right\|_{L^{2}}^{2} \\
+ & \frac{\varepsilon}{2}\left\|u-\tilde{u}_{N}\right\|_{H^{1}}^{2}+\frac{\varepsilon}{2}\left\|u-\hat{u}_{N}\right\|_{H^{1}}^{2} .
\end{aligned}
$$


Integrating (3.54) in time and applying the same technique as was used in (3.50) - (3.51), we see that

$$
\begin{aligned}
& \left\|\left(u-\tilde{u}_{N}\right)(t)\right\|_{L^{2}}^{2}+\varepsilon \int_{0}^{t}\left\|\left(u-\tilde{u}_{N}\right)(s)\right\|_{L^{2}}^{2} d s . \\
\leq & \kappa\left(h+h^{3}\right)+2 \int_{0}^{t}\left\|\left(u-\tilde{u}_{N}\right)(s)\right\|_{L^{2}} d s \\
\leq & \kappa h+2 \int_{0}^{1}\left\|\left(u-\tilde{u}_{N}\right)(s)\right\|_{L^{2}} d s .
\end{aligned}
$$

Applying the same Gronwall type argument we obtain

$$
\left\|u-\tilde{u}_{N}\right\|_{L^{\infty}\left(0, T ; L^{2}\right)} \leq \kappa\left(T, u_{0}, f\right) h^{\frac{1}{2}} .
$$

This completes the proof of the Theorem.

An under-resolved scheme with better error estimates (those comparable to the stationary case) can be derived by adding both $e^{-x / \varepsilon}$ and $x e^{-x / \varepsilon}$ into the basis.

THEOREM 3. Let $\tilde{\tilde{u}}_{N}$ be the solution of the modified finite element scheme

$$
\begin{aligned}
& \left(\frac{\partial \tilde{\tilde{u}}_{N}}{\partial t}, v\right)+\varepsilon\left(\tilde{\tilde{u}}_{N x}, v_{x}\right)-\left(\tilde{\tilde{u}}_{N x}, v\right)=(f, v), \forall v \in \tilde{\tilde{V}}_{N} \\
& \left(\left.\tilde{\tilde{u}}_{N}\right|_{t=0}, v\right)=\left(u_{0}, v\right), \forall v \in V_{N}
\end{aligned}
$$

where $\tilde{\tilde{u}}_{N} \in \tilde{\tilde{V}}_{N}=\operatorname{span}\left\{\varphi_{0}, \tilde{\varphi}_{0}, \varphi_{1}, \cdots, \varphi_{N-1}\right\}$ with $\tilde{\varphi}_{0}(x)=x\left(e^{-\frac{x}{\varepsilon}}-e^{-\frac{1}{\varepsilon}}\right) \sim x e^{-\frac{x}{\varepsilon}}$. Then there exists a constant $\kappa=\kappa\left(T, u_{0}, f\right)$ independent of $\varepsilon$ or $h$ such that

$$
\left\|u-\tilde{\tilde{u}}_{N}\right\|_{L^{\infty}\left(0, T ; L^{2}\right)} \leq \kappa\left(T, u_{0}, f\right)\left(\varepsilon^{\frac{1}{2}} h+\frac{h^{2}}{\varepsilon^{\frac{1}{2}}}\right) .
$$

Proof. Thanks to the systematic asymptotic expansion in the Appendix, we have

$$
\begin{aligned}
& \left\|u^{\epsilon}-c_{0}(t) \varphi_{0}(x)-\tilde{c}_{0}(t) \tilde{\varphi}_{0}(x)\right\|_{L^{\infty}\left(0, T ; H^{2}\right)} \\
\leq & \left\|u^{\epsilon}-c_{0}(t) e^{-\frac{x}{\varepsilon}}-\tilde{c}_{0}(t) x e^{-\frac{x}{\varepsilon}}\right\|_{L^{\infty}\left(0, T ; H^{2}\right)} \\
& \quad+\left\|c_{0}(t)\left(\left(1-e^{-\frac{1}{\varepsilon}}\right) x-1\right)+\tilde{c}_{0}(t) x e^{-\frac{1}{\varepsilon}}\right\|_{L^{\infty}\left(0, T ; H^{2}\right)} \\
\leq & \kappa .
\end{aligned}
$$

Combining this with the classical interpolation theory, we see that there exist constants $c_{1}(t), \cdots, c_{N-1}(t)$ such that

$$
\begin{aligned}
& \left\|u^{\varepsilon}-c_{0}(t) \varphi_{0}(x)-\tilde{c}_{0}(t) \tilde{\varphi}_{0}(x)-\sum_{j=1}^{N-1} c_{j}(t) \varphi_{j}\right\|_{L^{\infty}\left(0, T ; L^{2}\right)} \\
\leq & \kappa\left\|u^{\varepsilon}-c_{0}(t) \varphi_{0}(x)-\tilde{c}_{0}(t) \tilde{\varphi}_{0}(x)\right\|_{L^{\infty}\left(0, T ; H^{2}\right)} \cdot h^{2} \\
\leq & \kappa h^{2}, \\
& \left\|u^{\varepsilon}-c_{0}(t) \varphi_{0}(x)-\tilde{c}_{0}(t) \tilde{\varphi}_{0}(x)-\sum_{j=1}^{N-1} c_{j}(t) \varphi_{j}(x)\right\|_{L^{\infty}\left(0, T ; H^{1}\right)} \\
\leq & \kappa\left\|u^{\varepsilon}-c_{0}(t) \varphi_{0}(x)-\tilde{c}_{0}(t) \tilde{\varphi}_{0}(x)\right\|_{L^{\infty}\left(0, T ; H^{2}\right)} \cdot h \\
\leq & \kappa h .
\end{aligned}
$$


Denoting by $\hat{u}_{N}$ the expression

$$
\hat{u}_{N}=\sum_{j=0}^{N-1} c_{j}(t) \varphi_{j} \in \tilde{\tilde{V}}_{N}
$$

we can rewrite (3.61) - (3.62) as

$$
\begin{aligned}
\left\|u^{\varepsilon}-\hat{u}_{N}\right\|_{L^{\infty}\left(0, T ; L^{2}\right)} & \leq \kappa h^{2} \\
\left\|u^{\varepsilon}-\hat{u}_{N}\right\|_{L^{\infty}\left(0, T ; H^{1}\right)} & \leq \kappa h .
\end{aligned}
$$

By setting $v=\tilde{\tilde{u}}_{N}$ in (3.57), we see that

$$
\left\|\tilde{\tilde{u}}_{N}\right\|_{L^{\infty}\left(0, T ; L^{2}\right)} \leq \kappa .
$$

We now consider the difference of $u$ and $\tilde{\tilde{u}}_{N}$; we have

$$
\begin{gathered}
\left(\frac{\partial}{\partial t}\left(u-\tilde{\tilde{u}}_{N}\right), v\right)+\varepsilon\left(u_{x}-\tilde{\tilde{u}}_{N x}, v_{x}\right)-\left(u_{x}-\tilde{\tilde{u}}_{N x}, v\right)=0 \quad \forall v \in \tilde{\tilde{V}}_{N} \\
\left.\tilde{\tilde{u}}_{N}\right|_{t=0} \in V_{N},\left(\left.\tilde{\tilde{u}}_{N}\right|_{t=0}, v\right)=\left(u_{0}, v\right) \quad \forall v \in V_{N} .
\end{gathered}
$$

Equation (3.68) implies

$$
\left\|u_{0}-\left.\tilde{\tilde{u}}_{N}\right|_{t=0}\right\|_{L^{2}} \leq \kappa\left\|u_{0}\right\|_{H^{2}} \cdot h^{2} \leq \kappa h^{2} ;
$$

indeed, (3.68) can be written as

$$
\left(u_{0}-\left.\tilde{\tilde{u}}_{N}\right|_{t=0}, v\right)=0 \quad \forall v \in V_{N},\left.\tilde{u}_{N}\right|_{t=0} \in V_{N} .
$$

Hence, for every $\bar{u} \in V_{N}$,

$$
\begin{aligned}
& \left(u_{0}-\left.\tilde{\tilde{u}}_{N}\right|_{t=0}, u_{0}-\left.\tilde{\tilde{u}}_{N}\right|_{t=0}\right) \\
= & \left(u_{0}-\left.\tilde{\tilde{u}}_{N}\right|_{t=0}, u_{0}-\bar{u}\right)+\left(u_{0}-\left.\tilde{\tilde{u}}_{N}\right|_{t=0}, \bar{u}-\left.\tilde{\tilde{u}}_{N}\right|_{t=0}\right) \\
= & \left(u_{0}-\left.\tilde{\tilde{u}}_{N}\right|_{t=0}, u_{0}-\bar{u}\right) ;
\end{aligned}
$$

we thus have

$$
\left\|u_{0}-\left.\tilde{\tilde{u}}_{N}\right|_{t=0}\right\|_{L^{2}}^{2} \leq\left\|u_{0}-\left.\tilde{\tilde{u}}_{N}\right|_{t=0}\right\|_{L^{2}}\left\|u_{0}-\bar{u}\right\|_{L^{2}}, \quad \forall \bar{u} \in V_{N}
$$

and (3.69) follows from the usual interpolation inequalities.

Now notice that

$$
\begin{aligned}
& \text { 72) } \frac{1}{2} \frac{d}{d t}\left\|u-\tilde{\tilde{u}}_{N}\right\|_{L^{2}}^{2}+\varepsilon\left\|u_{x}-\tilde{\tilde{u}}_{N x}\right\|_{L^{2}}^{2} \\
& =\left(\frac{\partial}{\partial t}\left(u-\tilde{\tilde{u}}_{N}\right), u-\tilde{\tilde{u}}_{N}\right)+\varepsilon\left(u_{x}-\tilde{\tilde{u}}_{N x}, u_{x}-\tilde{\tilde{u}}_{N x}\right)-\left(u_{x}-\tilde{\tilde{u}}_{N x}, u-\tilde{\tilde{u}}_{N}\right) \\
& =\left(\frac{\partial}{\partial t}\left(u-\tilde{\tilde{u}}_{N}\right), \hat{u}_{N}-\tilde{\tilde{u}}_{N}\right)+\left(\frac{\partial}{\partial t}\left(u-\tilde{\tilde{u}}_{N}\right), u-\hat{u}_{N}\right) \\
& \quad \quad+\varepsilon\left(u_{x}-\tilde{\tilde{u}}_{N x}, \hat{u}_{N x}-\tilde{\tilde{u}}_{N x}\right)+\varepsilon\left(u_{x}-\tilde{\tilde{u}}_{N x}, u_{x}-\hat{u}_{N x}\right) \\
& \quad \quad-\left(u_{x}-\tilde{\tilde{u}}_{N x}, \hat{u}_{N}-\tilde{\tilde{u}}_{N}\right)-\left(u_{x}-\tilde{\tilde{u}}_{N x}, u-\hat{u}_{N}\right) \\
& =\left(\frac{\partial}{\partial t}\left(u-\tilde{\tilde{u}}_{N}\right), u-\hat{u}_{N}\right)+\varepsilon\left(u_{x}-\tilde{\tilde{u}}_{N x}, u_{x}-\hat{u}_{N x}\right)-\left(u_{x}-\tilde{\tilde{u}}_{N x}, u-\hat{u}_{N}\right)
\end{aligned}
$$




$$
\begin{aligned}
& \leq\left(\frac{\partial}{\partial t}\left(u-\tilde{\tilde{u}}_{N}\right), u-\hat{u}_{N}\right)+\varepsilon\left\|u-\tilde{\tilde{u}}_{N}\right\|_{H^{1}}\left\|u-\hat{u}_{N}\right\|_{H^{1}}+\left\|u-\tilde{\tilde{u}}_{N}\right\|_{H^{1}}\left\|u-\hat{u}_{N}\right\|_{L^{2}} \\
& \leq\left(\frac{\partial}{\partial t}\left(u-\tilde{\tilde{u}}_{N}\right), u-\hat{u}_{N}\right)+\frac{\varepsilon}{2}\left\|u-\tilde{\tilde{u}}_{N}\right\|_{H^{1}}^{2}+\kappa \varepsilon\left\|u-\hat{u}_{N}\right\|_{H^{1}}^{2}+\kappa \varepsilon^{-1}\left\|u-\hat{u}_{N}\right\|_{L^{2}}^{2} \\
& \leq\left(\frac{\partial}{\partial t}\left(u-\tilde{\tilde{u}}_{N}\right), u-\hat{u}_{N}\right)+\frac{\varepsilon}{2}\left\|u-\tilde{\tilde{u}}_{N}\right\|_{H^{1}}^{2}+\kappa\left(\varepsilon h+\frac{h^{3}}{\varepsilon}\right)
\end{aligned}
$$

(Thanks to (3.64) and (3.65)).

Hence, we have

$$
\frac{d}{d t}\left\|u-\tilde{\tilde{u}}_{N}\right\|_{L^{2}}^{2}+\varepsilon\left\|u-\tilde{\tilde{u}}_{N}\right\|_{H^{1}}^{2} \leq 2\left(\frac{\partial}{\partial t}\left(u-\tilde{\tilde{u}}_{N}\right), u-\hat{u}_{N}\right)+\kappa\left(\varepsilon h+\frac{h^{3}}{\varepsilon}\right) \text {. }
$$

Integrating (3.73) in time and utilizing (3.69), we find that

$$
\begin{aligned}
& \quad\left\|\left(u-\tilde{\tilde{u}}_{N}\right)(t)\right\|_{L^{2}}^{2}+\varepsilon \int_{0}^{t}\left\|\left(u-\tilde{\tilde{u}}_{N}\right)(s)\right\|_{H^{1}}^{2} d s \\
& \leq \kappa\left(h^{4}+\varepsilon h^{2}+\frac{h^{4}}{\varepsilon}\right)+2\left(\left(u-\tilde{\tilde{u}}_{N}\right)(t),\left(u-\hat{u}_{N}\right)(t)\right) \\
& \quad-2 \int_{0}^{t}\left(\left(u-\tilde{\tilde{u}}_{N}\right)(s),\left(\frac{\partial u}{\partial t}-\frac{\partial \hat{u}_{N}}{\partial t}\right)(s)\right) d s \\
& \leq \kappa\left(h^{2}+\varepsilon h^{2}+\frac{h^{4}}{\varepsilon}\right)+\frac{1}{2}\left\|\left(u-\tilde{\tilde{u}}_{N}\right)(t)\right\|_{L^{2}}^{2}+2\left\|\left(u-\hat{u}_{N}\right)(t)\right\|_{L^{2}}^{2} \\
& \quad+\frac{1}{2} \int_{0}^{t}\|(u-\tilde{\tilde{u}})(s)\|_{L^{2}}^{2} d s+2 \int_{0}^{t}\left\|\left(\frac{\partial u}{\partial t}-\frac{\partial \hat{u}_{N}}{\partial t}\right)(s)\right\|_{L^{2}}^{2} d s \\
& \leq \kappa\left(h^{4}+\varepsilon h^{2}+\frac{h^{4}}{\varepsilon}\right)+\frac{1}{2}\left\|\left(u-\tilde{\tilde{u}}_{N}\right)(t)\right\|_{L^{2}}^{2}+\frac{1}{2} \int_{0}^{t}\left(u-\tilde{\tilde{u}}_{N}\right)(s) \|_{L^{2}}^{2}
\end{aligned}
$$

(Thanks to (3.64) and our assumption on time derivative for the problem) .

This further implies

$$
\begin{aligned}
& \left\|\left(u-\tilde{\tilde{u}}_{N}\right)(t)\right\|_{L^{2}}^{2}+2 \varepsilon \int_{0}^{t}\left\|\left(u-\tilde{u}_{N}\right)(s)\right\|_{H^{1}}^{2} d s \\
& \leq \kappa\left(h^{4}+\varepsilon h^{2}+\frac{h^{4}}{\varepsilon}\right)+\int_{0}^{t}\left\|\left(u-\tilde{\tilde{u}}_{N}\right)(s)\right\|_{L^{2}}^{2} d s .
\end{aligned}
$$

Now we apply the usual Gronwall inequality . More precisely, we repeat the argument by setting

$$
g(t)=\int_{0}^{t}\left\|\left(u-\tilde{\tilde{u}}_{N}\right)(s)\right\|_{L^{2}}^{2} d s
$$

We then have

$$
g^{\prime}(t) \leq g(t)+\kappa\left(h^{4}+\varepsilon h^{2}+\frac{h^{4}}{\varepsilon}\right),
$$

or

$$
\frac{d}{d t}\left(e^{-t} g(t)\right) \leq \kappa\left(h^{4}+\varepsilon h^{2}+\frac{h^{4}}{\varepsilon}\right) .
$$


Hence

$$
g(t) \leq \kappa\left(T, u_{0}, f\right)\left(h^{4}+\varepsilon h^{2}+\frac{h^{4}}{\varepsilon}\right) \quad \forall t \in[0, T]
$$

Inserting this into (3.75) we find

$$
\left\|u-\tilde{\tilde{u}}_{N}\right\|_{L^{\infty}\left(0, T ; L^{2}\right)} \leq \kappa\left(T, u_{0}, f\right)\left(\varepsilon^{\frac{1}{2}} h+\frac{h^{2}}{\varepsilon^{\frac{1}{2}}}\right) .
$$

This indicates that the scheme (3.57) - (3.58) could capture the qualitative behavior in the under-resolved case $\left(h>>\varepsilon\right.$ ), as long as $h^{2} \varepsilon^{-\frac{1}{2}}<<1$ or $h<<\varepsilon^{\frac{1}{4}}$, (e.g. $h<<10^{-1}$ if $\varepsilon=10^{-4}$ ).

As for the case with one boundary layer base incorporated, we have an alternative way to derive the convergence rate for the under-resolved scheme as follows. Notice that

$$
\begin{aligned}
\left|\left(u_{x}-\tilde{\tilde{u}}_{N x}, u-\hat{u}_{N}\right)\right|= & \left|\left(u-\tilde{\tilde{u}}_{N}, u_{x}-\hat{u}_{N x}\right)\right| \\
\leq & \left\|u-\tilde{\tilde{u}}_{N}\right\|_{L^{2}}\left\|u_{x}-\hat{u}_{N x}\right\|_{L^{2}} \\
\leq & \frac{1}{4}\left\|u-\tilde{\tilde{u}}_{N}\right\|_{L^{2}}^{2}+\left\|u_{x}-\hat{u}_{N x}\right\|_{L^{2}}^{2} \\
\leq & \frac{1}{4}\left\|u-\tilde{\tilde{u}}_{N}\right\|_{L^{2}}^{2}+\kappa h^{2} \\
& (\text { Thanks to }(3.65)) .
\end{aligned}
$$

Hence we have,

82) $\frac{d}{d t}\left\|u-\tilde{\tilde{u}}_{N}\right\|_{L^{2}}^{2}+\varepsilon\left\|u-\tilde{\tilde{u}}_{N}\right\|_{H^{2}}^{2} \leq \kappa h^{2}+2\left(\frac{\partial}{\partial t}\left(u-\tilde{\tilde{u}}_{N}\right), u-\hat{u}_{N}\right)+\frac{1}{2}\left\|u-\tilde{\tilde{u}}_{N}\right\|_{L^{2}}^{2}$.

Integrating (3.82) in time and applying the same technique as was used in (3.74) - (3.75), we see that

$$
\begin{aligned}
& \left\|\left(u-\tilde{\tilde{u}}_{N}\right)(t)\right\|_{L^{2}}^{2}+2 \varepsilon \int_{0}^{t}\left\|\left(u-\tilde{u}_{N}\right)(s)\right\|_{L^{2}}^{2} d s . \\
\leq & \kappa\left(h^{2}+h^{3}+h^{4}\right)+2 \int_{0}^{t}\left\|\left(u-\tilde{\tilde{u}}_{N}\right)(s)\right\|_{L^{2}} d s \\
\leq & \kappa h^{2}+2 \int_{0}^{1}\left\|\left(u-\tilde{\tilde{u}}_{N}\right)(s)\right\|_{L^{2}} d s .
\end{aligned}
$$

Applying the same Gronwall type argument we obtain, as in (3.76) - (3.80)

$$
\left\|u-\tilde{\tilde{u}}_{N}\right\|_{L^{\infty}\left(0, T ; L^{2}\right)} \leq \kappa\left(T, u_{0}, f\right) h .
$$

Notice that the right hand side of (3.84) is independent of $\varepsilon$ which is in contrast to (3.80).

4. Numerical Results. The numerical calculations were carried out on an SGI Octane $225 \mathrm{Mhz}$ machine with $512 \mathrm{Mb}$ memory running IRIS 6.4. Figure 1 is for the equation

$$
\begin{aligned}
& \frac{\partial u}{\partial t}-\varepsilon u_{x x}-u_{x}=1 \\
& u(t, 0)=u(t, 1)=0 \\
& u^{0}(0, x)=x(1-x)
\end{aligned}
$$


with $\varepsilon=0.001$. Figure 2 is for the equation

$$
\begin{aligned}
& \frac{\partial u}{\partial t}-\varepsilon u_{x x}-u_{x}=t^{5} \\
& u(t, 0)=u(t, 1)=0, \\
& u^{0}(0, x)=x^{2}(1-x)^{6} .
\end{aligned}
$$

The grid size in space is 0.1 and it is 0.01 in time. The implicit Euler method is used for the time discretization. For our new scheme, when $T$ is fixed, we employ Gaussian elimination to solve the linear system $A x=b$ directly. This is possible since the associated matrix has the structure of a tridiagonal matrix plus an extra row and an extra column. What we observed is similar to the stationary case. The classical finite element method produces oscillations for coarse grid sizes in the space. We do not observe any oscillations with our new scheme. The results are shown in Figures 1 and 2. In both figures, $(a)$ and $\left(a^{\prime}\right)$ are approximate solutions at $T=1.0$ and $T=2.0$ using standard finite element methods respectively. Notice that there are oscillations; $(b)$ and $\left(b^{\prime}\right)$ are approximate solutions at $T=1.0$ and $T=2.0$ using the new scheme with one boundary layer base respectively. Notice that there is a sharp boundary layer near $x=0 ;(c)$ and $\left(c^{\prime}\right)$ are enlarged pictures of $(b)$ and $\left(b^{\prime}\right)$ near $x=0$ respectively.
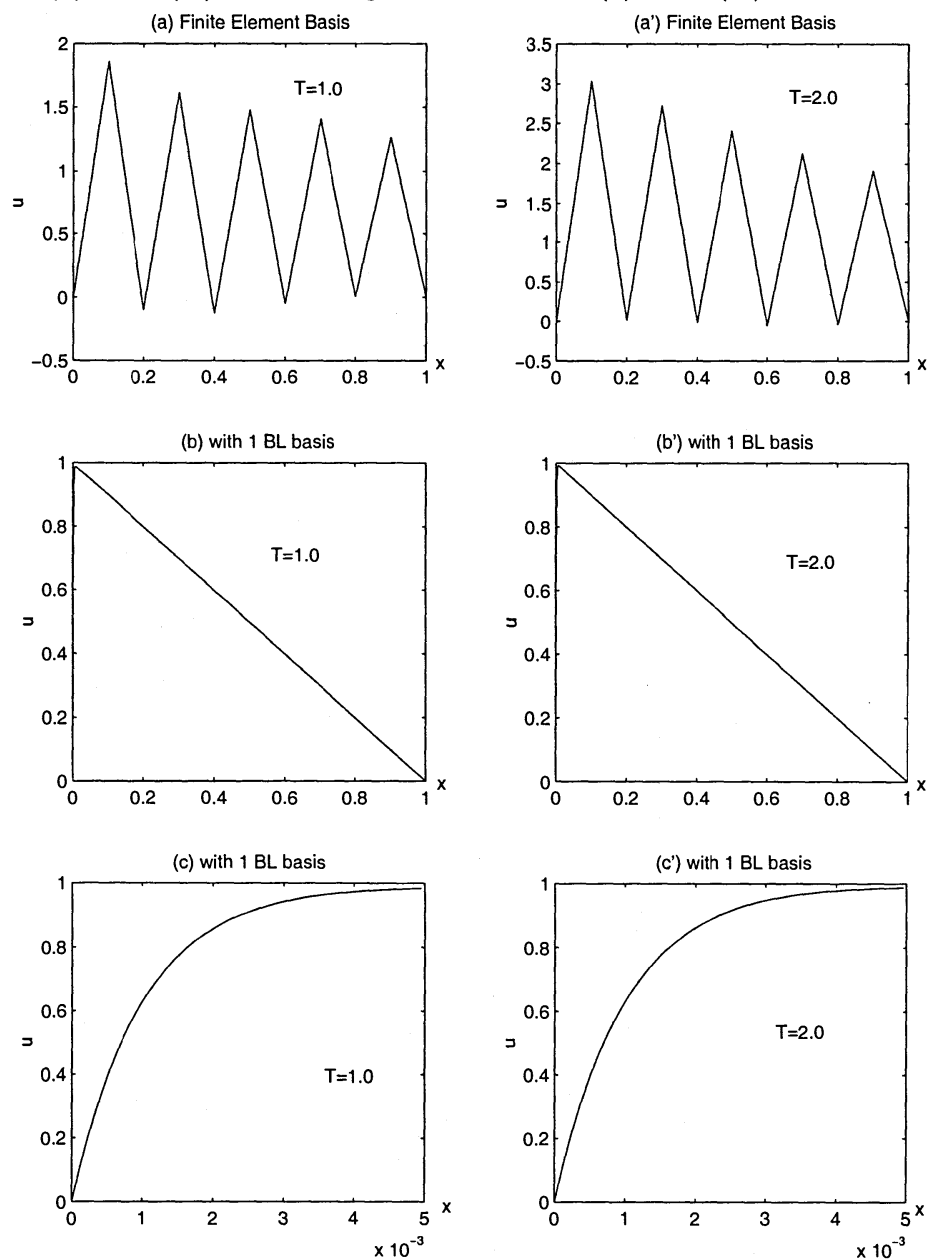

Figure 1: $u_{t}-\varepsilon u_{x x}-u_{x}=1, \varepsilon=10^{-3}, u^{0}(x)=x(1-x), N=10, \Delta t=0.01$ 

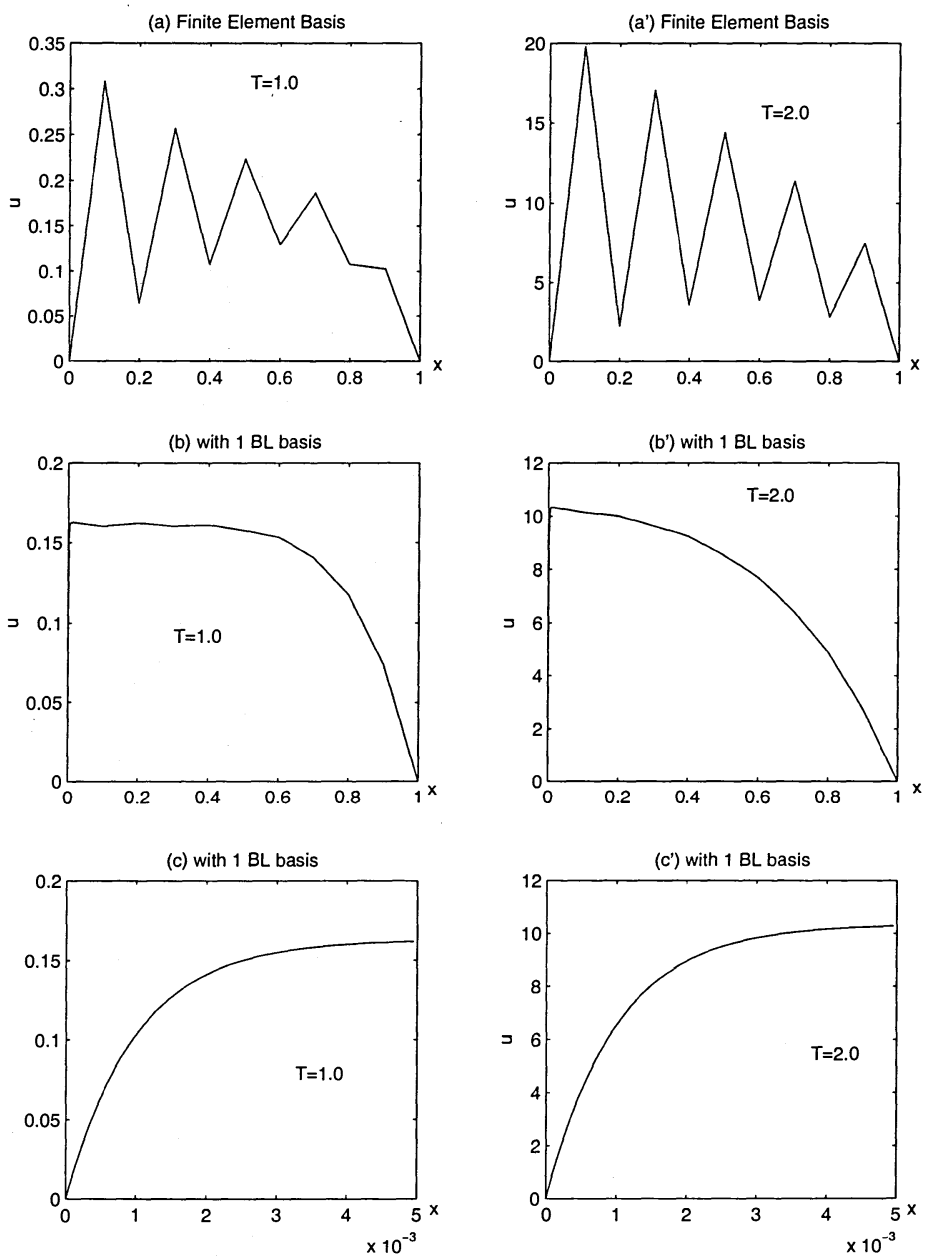

Figure 2: $u_{t}-\varepsilon u_{x x}-u_{x}=t^{5}, \varepsilon=10^{-3}, u^{0}(x)=x^{2}(1-x)^{6}, N=10, \Delta t=0.01$

5. Appendix: Systematic expansion for the model problem with a small diffusive coefficient. In this Appendix, we introduce and prove, in a systematic fashion, asymptotic expansions for the solutions to the following type of linear convection-diffusion equations

$$
\left\{\begin{array}{l}
\frac{\partial u^{\varepsilon}}{\partial t}-\varepsilon u_{x x}^{\varepsilon}-\beta u_{x}^{\varepsilon}=f \quad \text { in } \Omega \\
u^{\varepsilon}=u_{0} \quad \text { at } t=0 \\
u^{\varepsilon}=0 \quad \text { at } x=0 \text { and } L
\end{array}\right.
$$

for small diffusive coefficient $\varepsilon$. Here $\Omega$ is the interval $(0, L)$ in the one dimensional case. Extensions to higher dimensional cases do not raise any further difficulty, but will not be addressed here.

Two types of asymptotic expansions will be introduced. The first type is of the form

$$
u^{\varepsilon} \sim \sum_{j=0}^{\infty} \varepsilon^{j} u^{j}
$$


which is suitable in the interior of $\Omega$. This asymptotic expansion is not valid on $\bar{\Omega}$ in general due to the existence of a boundary layer. In order to develop an asymptotic expansion valid up to the boundary, correctors of various orders are needed to resolve the boundary layers of various orders. More specifically, we will have an asymptotic expansion of the form

$$
u^{\varepsilon} \sim \sum_{j=0}^{\infty} \varepsilon^{j}\left(u^{j}+\theta^{j}\right) .
$$

This outer expansion (outside the boundary layer) is suitable in $\bar{\Omega}$. The correctors $\theta^{j}$ are of the boundary layer type and their explicit form are needed in our design and analysis of the new schemes.

The problem we treat here is a prototype problem. The treatment we introduce here continues and generalizes the treatment by Vishik and Lyusternik (1957) and Lions (1973). The boundary layer analysis of other viscous perturbation of hyperbolic problems can be found in the work of Gisclon and Serre (1994), Grenier and Gues (1998) and Xin (1998) among others. Notice that we have a detailed analysis of the boundary layer for our model problem which was not found in other works. These detailed features are needed in the design and analysis of our numerical scheme. More precisely, we have to the leading order

$$
\theta^{j}=\sum_{k=0}^{j} c_{k} x^{k} e^{-\beta x / \varepsilon} .
$$

The first two terms in the expansion are useful in the numerical analysis of our underresolved scheme with a boundary layer base.

Asymptotic expansion in the interior. In this section, we study the asymptotic behavior of the solutions to the following one dimensional linear convectiondiffusion equation with a small diffusive coefficient:

$$
\begin{aligned}
& \frac{\partial u^{\varepsilon}}{\partial t}-\varepsilon u_{x x}^{\varepsilon}-\beta u_{x}^{\varepsilon}=f \text { in }(0, L) \times(0, \infty), \\
& u^{\varepsilon}=u_{0} \text { at } t=0 \\
& u^{\varepsilon}=0 \text { at } x=0 \text { and } L
\end{aligned}
$$

where $\beta$ is a fixed positive constant. The extension to variable non-vanishing $\beta$ 's does not raise any major difficulty.

We first consider an expansion of $u$ in a power series in $\varepsilon$ of the form,

$$
u^{\varepsilon} \sim \sum_{j=0}^{\infty} \varepsilon^{j} u^{j}(t, x) .
$$

Inserting this into the equation we obtain

$$
\sum_{j=0}^{\infty} \varepsilon^{j} \frac{\partial u^{j}}{\partial t}(t, x)-\sum_{j=0}^{\infty} \varepsilon^{j+1} u_{x x}^{j}(t, x)-\beta \sum_{j=0}^{\infty} \varepsilon^{j} u_{x}^{j}(t, x)=f .
$$

By formal identification of the coefficients of the powers of $\varepsilon$ we obtain,

$$
\frac{\partial u^{0}}{\partial t}-\beta u_{x}^{0}=f
$$


and

$$
\frac{\partial u^{j}}{\partial t}-\beta u_{x}^{j}=u_{x x}^{j-1}, \quad \text { for } j \geq 1 .
$$

These are linear transport equations which can be solved recursively using the method of characteristics (see for instance F. John (1982)). In fact the projected characteristic takes the form

$$
x+\beta t=c .
$$

Hence the natural boundary condition for each of them is the upwind boundary condition,

$$
u^{j}=0 \text { at } L,
$$

and the natural initial conditions are

$$
\begin{aligned}
& u^{0}=u_{0} \text { at } t=0, \\
& u^{j}=0 \text { at } t=0, \text { for } j \geq 1 .
\end{aligned}
$$

These equations can be solved explicitly.

Certain compatibility conditions are needed to ensure the smoothness of these solutions $u^{j}$. More precisely in order to ensure that $u^{0} \in C^{n}$ we need

$$
u_{0}, f \in C^{n}
$$

and

$$
\begin{aligned}
0 & =\frac{\partial^{l}}{\partial t^{l}} u^{0}(L, 0) \\
& =\beta^{l} \frac{d^{l}}{d x^{l}} u_{0}(L)+\sum_{k=0}^{l-1} \beta^{k} \frac{\partial^{l-1}}{\partial t^{l-1-k} \partial x^{k}} f(L, 0)
\end{aligned}
$$

for all $l \leq n$. In order to ensure that $u^{j} \in C^{n}, j \geq 1$ we need

$$
u_{x x}^{j-1} \in C^{n}, \text { or } u_{0}, f \in C^{n+2 j}
$$

and

$$
0=\frac{\partial^{l}}{\partial t^{l}} u^{j}(L, 0)=\sum_{k=0}^{l-1} \beta^{k} \frac{\partial^{l-1}}{\partial t^{l-1-k} \partial x^{k}} u_{x x}^{j-1}(L, 0)
$$

for all $l \leq n$.

A general treatment of the compatibility conditions for nonlinear problems can be found in the work of Temam (1982).

Convergence of the asymptotic expansion (5.6) to all orders, in $L^{\infty}\left(0, T ; L^{2}(\Omega)\right)$, can not be proven (directly) at this stage, but will follow from subsequent results of asymptotic expanison valid up to the boundary and the asymptotic formular for the boundary layer functions. 
Asymptotic expansion up to the boundary. In order to figure out the form of boundary layer type functions, we use a standard stretched coordinate argument in the boundary layer. Assuming that

$$
u^{\varepsilon} \sim \sum_{j=0}^{\infty} \varepsilon^{j}\left(u^{j}(t, x)+\theta^{j}\left(t, \frac{x}{\varepsilon^{\alpha}}\right)\right)
$$

and substituting into the equation (5.3), we deduce that, up to the leading order,

$$
-\varepsilon^{1-2 \alpha} \frac{\partial^{2} \theta^{0}}{\partial X^{2}}-\beta \varepsilon^{-\alpha} \frac{\partial \theta^{0}}{\partial X}=0
$$

In order to balance these two terms, we choose

$$
\alpha=1 \text {. }
$$

Hence (5.15) can be written as:

$$
u^{\varepsilon}(t, x) \sim \sum_{j=0}^{\infty} \varepsilon^{j}\left(u^{j}(t, x)+\theta^{j}\left(t, \frac{\beta x}{\varepsilon}\right)\right) .
$$

Inserting (5.17) into (5.3), denoting $\frac{\beta x}{\varepsilon}=X^{1}$, we obtain

$$
\sum_{j=0}^{\infty}\left(\varepsilon^{j} \frac{\partial u^{j}}{\partial t}-\varepsilon^{j+1} u_{x x}^{j}-\beta \varepsilon^{j} \frac{\partial u^{j}}{\partial x}+\varepsilon^{j} \frac{\partial \theta^{j}}{\partial t}-\beta^{2} \varepsilon^{j-1} \theta_{X X}^{j}-\beta^{2} \varepsilon^{j-1} \theta_{X}^{j}\right)=f
$$

Collecting the terms of the same order in $\varepsilon$ and utilizing the equations for $u^{j}$, we obtain,

$$
\begin{aligned}
& -\frac{\partial^{2} \theta^{0}}{\partial X^{2}}-\frac{\partial \theta^{0}}{\partial X}=0, \\
& -\frac{\partial^{2} \theta^{j}}{\partial X^{2}}-\frac{\partial \theta^{j}}{\partial X}=-\frac{1}{\beta^{2}} \frac{\partial \theta^{j-1}}{\partial t} \quad \text { for } j \geq 1 .
\end{aligned}
$$

The boundary conditions for the correctors $\theta^{j}$ are:

$$
\begin{gathered}
\theta^{j}=0 \text { at } x=L, \\
\theta^{j}=-u^{j} \text { at } x=0,
\end{gathered}
$$

since we need homogeneous boundary conditions for $u^{j}+\theta^{j}$.

Notice that

$$
\theta^{0}=0 \text { at } t=0
$$

since $u^{0}=u_{0}=0$ at $x=0, t=0$, which is a compatibility condition that we assume. Indeed, we can prove a more general result regarding $\theta^{j}$.

Proposition 4. For fixed $k>0$ we have

$$
\theta^{j}=0 \text { at } t=0 \text { for } j \leq k,
$$

\footnotetext{
${ }^{1}$ We use $\frac{\beta x}{\varepsilon}$ as the stretched coordinate because it is a non-dimensional number. It is also related to the Reynolds number in the fluid dynamic case.
} 
provided the following compatibility condition holds

$$
\frac{\partial^{l} u^{j}}{\partial t^{l}}(0,0)=0 \quad \text { for } l+j \leq k .
$$

Proof. We will prove a stronger result, namely

$$
\frac{\partial^{l} \theta^{j}}{\partial t^{l}}=0 \text { at } t=0 \text { for } l+j \leq k \text {. }
$$

Notice that (5.23) is a special case of (5.25) with $l=0$.

In order to prove (5.25), we perform induction on $j$ and $l$. For $j=0$, we notice that $\frac{\partial^{l} \theta^{0}}{\partial t^{l}}$ satisfies

$$
\begin{aligned}
& -\varepsilon \frac{\partial^{2}}{\partial x^{2}}\left(\frac{\partial^{l} \theta^{0}}{\partial t^{l}}\right)-\beta \frac{\partial}{\partial x}\left(\frac{\partial^{l} \theta^{0}}{\partial t^{l}}\right)=0, \\
& \frac{\partial^{l} \theta^{0}}{\partial t^{l}}=0 \text { at } x=L, \frac{\partial^{l} \theta^{0}}{\partial t^{l}}=-\frac{\partial^{l} u^{0}}{\partial t^{l}} \text { at } x=0 .
\end{aligned}
$$

At $t=0$, we have $\frac{\partial^{l} \theta^{0}}{\partial t^{l}}(0,0)=0$ for $l \leq k$ by the compatibility assumption (5.24). Hence

$$
\frac{\partial^{l} \theta^{0}}{\partial t^{l}}=0 \text { at } t=0 \text { for } l \leq k
$$

by a simple elliptic uniqueness result. Now suppose that for $0 \leq j_{0}<k$, we have

$$
\frac{\partial^{l} \theta^{j_{0}}}{\partial t^{l}} \text { at } t=0 \text { for } l+j_{0} \leq k
$$

Then $\frac{\partial^{l-1}}{\partial t^{l-1}} \theta^{j_{0}+1}$ satisfies the following equation, thanks to (5.19), (5.20) and (5.21),

$$
\begin{aligned}
& -\varepsilon \frac{\partial^{2}}{\partial x^{2}}\left(\frac{\partial^{l-1}}{\partial t^{l-1}} \theta^{j_{0}+1}\right)-\beta \frac{\partial}{\partial x}\left(\frac{\partial^{l-1}}{\partial t^{l-1}} \theta^{j_{0}+1}\right)=-\frac{1}{\varepsilon} \frac{\partial^{l}}{\partial t^{l}} \theta^{j_{0}} \\
& \frac{\partial^{l-1}}{\partial t^{l-1}} \theta^{j_{0}+1}=0 \text { at } x=L, \frac{\partial^{l-1}}{\partial t^{l-1}} \theta^{j_{0}+1}=-\frac{\partial^{l-1} u^{j_{0}+1}}{\partial t^{l-1}} \text { at } x=0,
\end{aligned}
$$

Combining (5.29), (5.30), (5.31) and (5.24), we obtain

$$
\frac{\partial^{j-1}}{\partial t^{l-1}} \theta^{j_{0}+1}=0 \text { at } t=0 \text { for } l+j_{0} \leq k,
$$

thanks to the standard elliptic uniqueness result. With this, we end the proof of the proposition.

With this proposition, we can prove rigorously the validity of the expansion (5.17) using standard energy estimates.

THEOREM 5. Assume that the data are smooth and that they satisfy compatibility conditions (5.13), (5.14) and (5.24) and set

$$
w_{k}^{\varepsilon}=u^{\varepsilon}-\sum_{j=0}^{k} \varepsilon^{j}\left(u^{j}(t, x)+\theta^{j}\left(t, \frac{\beta x}{\varepsilon}\right)\right) .
$$


Then there exists a constant $\kappa$ independent of $\varepsilon$ ( but depending on $L, u_{0}, f$ and $k$ ) such that

$$
\begin{aligned}
& \left\|w_{k}^{\varepsilon}\right\|_{L^{\infty}\left(0, T ; L^{2}\right)} \leq \kappa \varepsilon^{k+1}, \\
& \left\|w_{k}^{\varepsilon}\right\|_{L^{2}\left(0, T ; H^{1}\right)} \leq \kappa \varepsilon^{k+\frac{1}{2}}, \\
& \left\|w_{k}^{\varepsilon}\right\|_{L^{\infty}\left(0, T ; H^{1}\right)} \leq \kappa \varepsilon^{k} \\
& \left\|w_{k}^{\varepsilon}\right\|_{L^{2}\left(0, T ; H^{2}\right)} \leq \kappa \varepsilon^{k-\frac{1}{2}}, \\
& \left\|w_{k}^{\varepsilon}\right\|_{L^{\infty}\left(0, T ; H^{2}\right)} \leq \kappa \varepsilon^{k-1} \\
& \left\|w_{k}^{\varepsilon}\right\|_{L^{\infty}((0, T) \times(0, L))} \leq \kappa \varepsilon^{k+\frac{1}{2}} .
\end{aligned}
$$

Proof. It is easy to see that $w_{k}^{\varepsilon}$ satisfies the following equation and boundary and initial conditions:

$$
\begin{aligned}
& \frac{\partial w_{k}^{\varepsilon}}{\partial t}-\varepsilon w_{k x x}^{\varepsilon}-\beta w_{k x}^{\varepsilon}=\varepsilon^{k+1} u_{x x}^{k}-\varepsilon^{k} \frac{\partial \theta^{k}}{\partial t}, \\
& w_{k}^{\varepsilon}=0 \text { at } t=0, \\
& w_{k}^{\varepsilon}=0 \text { at } x=0, L,
\end{aligned}
$$

thanks to (5.8), (5.9), (5.10), (5.11), (5.12), (5.19), (5.19), (5.20), (5.21) and (5.23).

Multiplying (5.39) by $w_{k}^{\varepsilon}$ and integrating over $\Omega$, we obtain:

$$
\begin{aligned}
& \frac{1}{2} \frac{d}{d t}\left\|w_{k}^{\varepsilon}\right\|_{L^{2}}^{2}+\varepsilon\left\|w_{k x}^{\varepsilon}\right\|_{L^{2}}^{2} \\
\leq & \varepsilon^{k+1}\left\|u_{x x}^{k}\right\|_{L^{2}}\left\|w_{k}^{\varepsilon}\right\|_{L^{2}}+\varepsilon^{k}\left\|x \frac{\partial \theta^{k}}{\partial t}\right\|_{L^{2}}\left\|\frac{w_{k}^{\varepsilon}}{x}\right\|_{L^{2}}, \\
\leq & \frac{1}{2}\left\|w_{k}^{\varepsilon}\right\|_{L^{2}}^{2}+\frac{1}{2} \varepsilon^{2 k+2}\left\|u_{x x}^{k}\right\|_{L^{2}}^{2}+\varepsilon^{k}\left\|x \frac{\partial \theta^{k}}{\partial t}\right\|_{L^{2}}\left\|w_{k x}^{\varepsilon}\right\|_{L^{2}}, \\
\leq & \frac{1}{2}\left\|w_{k}^{\varepsilon}\right\|_{L^{2}}^{2}+\frac{\varepsilon}{2}\left\|w_{k x}^{\varepsilon}\right\|_{L^{2}}^{2}+\frac{1}{2} \varepsilon^{2 k+2}\left\|u_{x x}^{k}\right\|_{L^{2}}^{2}+\frac{\varepsilon^{2 k-1}}{2}\left\|x \frac{\partial \theta^{k}}{\partial t}\right\|_{L^{2}}^{2}, \\
\leq & \frac{1}{2}\left\|w_{k}^{\varepsilon}\right\|_{L^{2}}^{2}+\frac{\varepsilon}{2}\left\|w_{k x}^{\varepsilon}\right\|_{L^{2}}^{2}+\kappa \varepsilon^{2 k+2}
\end{aligned}
$$

where we have used the approximate form of $\theta^{\varepsilon}$ developed in the next theorem whose proof is independent of the proof of this theorem. We also used the classical Hardy inequality. Hence we have,

$$
\frac{d}{d t}\left\|w_{k}^{\varepsilon}\right\|_{L^{2}}^{2}+\varepsilon\left\|w_{k x}^{\varepsilon}\right\|_{L^{2}}^{2} \leq\left\|w_{k}^{\varepsilon}\right\|_{L^{2}}^{2}+2 \kappa \varepsilon^{2 k+2} .
$$

Applying the usual Gronwall inequality and using (5.40), we obtain (5.33) and (5.34).

To derive (5.35) and (5.36) (which are needed for the numerical analysis of our approximation scheme for (5.1)), we multiply (5.39) by $-w_{k x x}^{\varepsilon}$ and integrate over $\Omega=(0, L)$. Notice that

$$
\begin{aligned}
\int_{\Omega} \frac{\partial w_{k}^{\varepsilon}}{\partial t}\left(-w_{k x x}^{\varepsilon}\right) & =\frac{1}{2} \frac{d}{d t}\left\|w_{k x}^{\varepsilon}\right\|_{L^{2}}^{2} \\
\int_{\Omega}-\varepsilon w_{k x x}^{\varepsilon}\left(-w_{k x x}^{\varepsilon}\right) & =\varepsilon\left\|w_{k x x}^{\varepsilon}\right\|_{L^{2}}^{2},
\end{aligned}
$$




$$
\begin{aligned}
\left\|\beta \int_{\Omega} w_{k x}^{\varepsilon} w_{k x x}^{\varepsilon}\right\| & \leq \beta\left\|w_{k x x}^{\varepsilon}\right\|_{L^{2}}\left\|w_{k x}^{\varepsilon}\right\|_{L 2}, \\
& \leq \frac{\varepsilon}{4}\left\|w_{k x x}^{\varepsilon}\right\|_{L^{2}}^{2}+\frac{\beta^{2}}{\varepsilon}\left\|w_{k x}^{\varepsilon}\right\|_{L^{2}}^{2}, \\
\left\|\int_{\Omega} \varepsilon^{k+1} u_{x x}^{k} w_{k x x}^{\varepsilon}\right\| & \leq \varepsilon^{k+1}\left\|u_{x x}^{k}\right\|_{L^{2}}\left\|w_{k x x}^{\varepsilon}\right\|_{L^{2}}, \\
& \leq \frac{\varepsilon}{4}\left\|w_{k x x}^{\varepsilon}\right\|_{L^{2}}^{2}+\varepsilon^{2 k+1}\left\|u_{x x}^{k}\right\|_{L^{2}}^{2} \\
\left\|\int_{\Omega} \varepsilon^{k} \frac{\partial \theta^{k}}{\partial t} w_{k x x}^{\varepsilon}\right\| & \leq \varepsilon^{k}\left\|\frac{\partial \theta^{k}}{\partial t}\right\|_{L^{2}}\left\|w_{k x}^{\varepsilon}\right\|_{L^{2}} \\
& \leq \frac{\varepsilon}{4}\left\|w_{k x x}^{\varepsilon}\right\|_{L^{2}}^{2}+\varepsilon^{2 k-1}\left\|\frac{\partial \theta^{k}}{\partial t}\right\|_{L^{2}}^{2} .
\end{aligned}
$$

Thus we have

$$
\begin{aligned}
& \frac{1}{2} \frac{d}{d t}\left\|w_{k x}^{\varepsilon}\right\|_{L^{2}}^{2}+\varepsilon\left\|w_{k x x}^{\varepsilon}\right\|_{L^{2}}^{2} \\
\leq & \frac{3}{4} \varepsilon\left\|w_{k x x}^{\varepsilon}\right\|_{L^{2}}^{2}+\frac{\beta^{2}}{\varepsilon}\left\|w_{k x}^{\varepsilon}\right\|_{L^{2}}^{2}+\varepsilon^{2 k+1}\left\|u_{x x}^{k}\right\|_{L^{2}}^{2}+\varepsilon^{2 k-1}\left\|\frac{\partial \theta^{k}}{\partial t}\right\|_{L^{2}}^{2},
\end{aligned}
$$

or

$$
\frac{d}{d t}\left\|w_{k x}^{\varepsilon}\right\|_{L^{2}}^{2}+\frac{\varepsilon}{2}\left\|w_{k x x}^{\varepsilon}\right\|_{L^{2}}^{2} \leq \frac{2 \beta^{2}}{\varepsilon}\left\|w_{k x}^{\varepsilon}\right\|_{L^{2}}^{2}+2 \varepsilon^{2 k+1}\left\|u_{x x}^{k}\right\|_{L^{2}}^{2}+2 \varepsilon^{2 k-1}\left\|\frac{\partial \theta^{k}}{\partial t}\right\|_{L^{2}}^{2}
$$

Integrating (5.48) over $(0, t)$ and utilizing the estimate (5.34), we obtain (5.35) and (5.36). Lastly, we notice that (5.38) is a consequence of (5.33) and (5.35). Indeed, thanks to Agmon's inequality, we have

$$
\begin{aligned}
\left\|w_{k}^{\varepsilon}\right\|_{L^{\infty}((0, T) \times \Omega)} & \leq \sqrt{2}\left\|w_{k}^{\varepsilon}\right\|_{L^{\infty}\left(0, T ; L^{2}\right)}^{\frac{1}{2}}\left\|w_{k x}^{\varepsilon}\right\|_{L^{\infty}\left(0, T ; L^{2}\right)}^{\frac{1}{2}} \\
& \leq \kappa \varepsilon^{k+\frac{1}{2}}, \forall t \in[0, T] .
\end{aligned}
$$

This implies (5.38).

In order to derive $L^{\infty}\left(0, T ; H^{2}\right)$ estimates we first derive $L^{\infty}\left(0, T ; L^{2}\right)$ estimates on the time derivative and then apply elliptic estimates. For this purpose, we take the time derivative of the equation satisfied by $w_{k}^{\varepsilon}(5.39)$ and obtain

$$
\begin{aligned}
& \frac{\partial^{2} w_{k}^{\varepsilon}}{\partial t^{2}}-\varepsilon\left(\frac{\partial w_{k}^{\varepsilon}}{\partial t}\right)_{x x}-\beta\left(\frac{\partial w_{k}^{\varepsilon}}{\partial t}\right)_{x}=\varepsilon^{k+1} \Delta \frac{\partial u^{k}}{\partial t}-\varepsilon^{k} \frac{\partial^{2} \theta^{k}}{\partial t^{2}} \\
& \frac{\partial w_{k}^{\varepsilon}}{\partial t}=0 \text { at } x=0, L \\
& \frac{\partial w_{k}^{\varepsilon}}{\partial t}=0 \text { at } t=0 .
\end{aligned}
$$

(The zero initial condition is due to the equation and the fact that $w_{k}^{\varepsilon}=0$ at $t=$ $0, u^{k}=0$ at $t=0$ and $\frac{\partial \theta^{k}}{\partial t}=0$ at $t=0$.)

We multiply the equation for $\frac{\partial w_{k}^{\varepsilon}}{\partial t}$ by $\frac{\partial w_{k}^{\varepsilon}}{\partial t}$, integrate over $\Omega$, and utilize the following estimates:

$$
\int_{\Omega}-\varepsilon\left(\frac{\partial w_{k}^{\varepsilon}}{\partial t}\right)_{x x} \frac{\partial w_{k}^{\varepsilon}}{\partial t}=\varepsilon\left\|\frac{\partial^{2} w_{k}^{\varepsilon}}{\partial t \partial x}\right\|_{L^{2}}^{2}
$$




$$
\begin{aligned}
\int_{\Omega}-\beta\left(w_{k}^{\varepsilon}\right)_{x} w_{k}^{\varepsilon} & =0 \\
\int_{\Omega} \varepsilon^{k+1} \Delta \frac{\partial u^{k}}{\partial t} \frac{\partial w_{k}^{\varepsilon}}{\partial t} & \leq \varepsilon^{k+1}\left\|\Delta \frac{\partial u^{k}}{\partial t}\right\|_{L^{2}}\left\|\frac{\partial w_{k}^{\varepsilon}}{\partial t}\right\|_{L^{2}} \\
& \leq \frac{1}{4}\left\|\frac{\partial w_{k}^{\varepsilon}}{\partial t}\right\|_{L^{2}}^{2}+\varepsilon^{2 k+2}\left\|\Delta \frac{\partial u^{k}}{\partial t}\right\|_{L^{2}}^{2}, \\
\int_{\Omega}-\varepsilon^{k} \frac{\partial^{2} \theta^{k}}{\partial t^{2}} \frac{\partial w_{k}^{\varepsilon}}{\partial t} & \leq \varepsilon^{k}\left\|x \frac{\partial^{2} \theta^{k}}{\partial t^{2}}\right\|_{L^{2}}\left\|\frac{1}{x} \frac{\partial w_{k}^{\varepsilon}}{\partial t}\right\|_{L^{2}} \\
& \leq \varepsilon^{k}\left\|\frac{\partial^{2} w_{k}^{\varepsilon}}{\partial t \partial x}\right\|_{L^{2}}\left\|x \frac{\partial^{2} \theta^{k}}{\partial t^{2}}\right\|_{L^{2}} \\
& \leq \frac{\varepsilon}{2}\left\|\frac{\partial^{2} w_{k}^{\varepsilon}}{\partial t \partial x}\right\|_{L^{2}}+\frac{\varepsilon^{2 k-1}}{2}\left\|x \frac{\partial^{2} \theta^{k}}{\partial t^{2}}\right\|_{L^{2}}^{2} \\
& \leq \frac{\varepsilon}{2}\left\|\frac{\partial^{2} w_{k}^{\varepsilon}}{\partial t \partial x}\right\|_{L^{2}}^{2}+\kappa \varepsilon^{2 k+2}
\end{aligned}
$$

We then find

$$
\frac{d}{d t}\left\|\frac{\partial w_{k}^{\varepsilon}}{\partial t}\right\|_{L^{2}}^{2}+\varepsilon\left\|\frac{\partial w_{k}^{\varepsilon}}{\partial t \partial x}\right\|_{L^{2}}^{2} \leq \frac{1}{2}\left\|\frac{\partial w_{k}^{\varepsilon}}{\partial t}\right\|_{L^{2}}+\kappa \varepsilon^{2 k+2} .
$$

Thus the usual Gronwall inequality implies

$$
\begin{aligned}
\left\|\frac{\partial w_{k}^{\varepsilon}}{\partial t}\right\|_{L^{\infty}\left(0, T ; L^{2}\right)} & \leq \kappa \varepsilon^{k+1} \\
\left\|\frac{\partial w_{k}^{\varepsilon}}{\partial t}\right\|_{L^{2}\left(0, T ; H^{1}\right)} & \leq \kappa \varepsilon^{k+\frac{1}{2}} .
\end{aligned}
$$

Next we rewrite the equation for $w_{k}^{\varepsilon}$ as:

$$
\begin{aligned}
& -\varepsilon w_{k x x}^{\varepsilon}=\varepsilon^{k+1} \Delta u^{k}-\varepsilon^{k} \frac{\partial \theta^{k}}{\partial t}-\frac{\partial w_{k}^{\varepsilon}}{\partial t}+\beta w_{k x}^{\varepsilon} \\
& w_{k}^{\varepsilon}=0 \text { at } x=0, L
\end{aligned}
$$

We then deduce from elliptic theory

$$
\begin{aligned}
\left\|w_{k}^{\varepsilon}\right\|_{L^{\infty}\left(0, T ; H^{2}\right)} \leq & \frac{1}{\varepsilon}\left(\varepsilon^{k+1}\left\|\Delta u^{k}\right\|_{L^{\infty}\left(0, T ; L^{2}\right)}+\varepsilon^{k}\left\|\frac{\partial \theta^{k}}{\partial t}\right\|_{L^{\infty}\left(0, T ; L^{2}\right)}\right. \\
& \left.+\left\|\frac{\partial w_{k}^{\varepsilon}}{\partial t}\right\|_{L^{\infty}\left(0, T ; L^{2}\right)}+\beta\left\|w_{k}^{\varepsilon}\right\|_{L^{\infty}\left(0, T ; H^{1}\right)}\right) \\
\leq & \frac{\kappa}{\varepsilon}\left(\varepsilon^{k+1}+\varepsilon^{k+\frac{1}{2}}+\varepsilon^{k+\frac{1}{2}}+\varepsilon^{k}\right) \\
\leq & \kappa \varepsilon^{k-1} .
\end{aligned}
$$

This completes the proof of Theorem 5 .

REMARK. A useful observation is that we have the same estimate for $w_{k}^{\varepsilon}$ as for the $\frac{\partial w_{k}^{\varepsilon}}{\partial t}$, at least for the $L^{\infty}\left(0, T ; L^{2}\right)$ and $L^{2}\left(0, T ; H^{1}\right)$ norms, provided suitable compatibility conditions are satisfied (see (5.13), (5.14), (5.24)). In fact we can derive the same kind of estimates for $\frac{\partial w_{k}^{\varepsilon}}{\partial t}$ in other spaces (i.e. $\left.L^{\infty}\left(0, T ; H^{1}\right), L^{\infty}\left(0, T ; H^{2}\right)\right)$ as those for $w_{k}^{\varepsilon}$ provided that higher order compatibility conditions are satisfied. This is used in our numerical analysis. 
Our last objective in this section is to demonstrate that the $\theta^{j}$ 's are real boundary layer type functions, i.e., they decay rapidly as $x$ increases. We shall fulfill this task by deriving an asymptotic formula for $\theta^{j}$.

First, $\theta^{0}$ can be calculated explicitly by using (5.19) and (5.21); we have

$$
\theta^{0}(t, x)=-u^{0}(t, 0) \frac{e^{-\beta x / \varepsilon}-e^{-\beta L / \varepsilon}}{1-e^{-\beta L / \varepsilon}}
$$

Thus asymptotically we have

$$
\theta^{0}(t, x)=-u^{0}(t, x) e^{-\beta x / \varepsilon}+e . s . t .,
$$

where e.s.t. represents terms exponentially small in any Sobolev norm in time variable $t$ and space variable $x$. More precisely, we have:

Definition. A function $g^{\varepsilon}$ is called an exponentially small term, denoted e.s.t., if there exists $\alpha \in(0,1)$ such that for any $k, l \geq 0$, there exists a constant $c_{\alpha, k, l}$,

$$
\left\|g^{\varepsilon}\right\|_{H^{k}\left(0, T ; H^{l}(\Omega)\right)} \leq c_{\alpha, k, l} e^{-\frac{\alpha \beta L}{\varepsilon}} .
$$

Then $\theta^{1}$ can be calculated explicitly using (5.19), (5.21) and (5.49):

$$
\begin{aligned}
\theta^{1}(t, x)= & -u^{1}(t, 0) \frac{e^{-\beta x / \varepsilon}-e^{-\beta L / \varepsilon}}{1-e^{-\beta L / \varepsilon}} \\
& +\frac{\partial u^{0}(t, 0)}{\partial t} \frac{e^{-\beta L / \varepsilon}}{\varepsilon \beta\left(1-e^{-\beta L / \varepsilon}\right)}\left(L \frac{e^{-\beta x / \varepsilon}-e^{-\beta L / \varepsilon}}{1-e^{-\beta L / \varepsilon}}-(L-x)\right) \\
& +\frac{x}{\varepsilon \beta} e^{-\beta x / \varepsilon} \frac{\partial u^{0}(t, 0)}{\partial t} \frac{1}{1-e^{-\beta L / \varepsilon}}
\end{aligned}
$$

or asymptotically,

$$
\theta^{1}(t, x)=\frac{x}{\varepsilon \beta} e^{-\beta x / \varepsilon} \frac{\partial u^{0}(t, 0)}{\partial t}-e^{-\beta x / \varepsilon} u^{1}(t, 0)+e . s . t .
$$

The method can be generalized by induction to $\theta^{j}$ for all $j \geq 2$. Indeed we have

THEOREM 6. Assuming that the data are smooth and satisfy compatibility conditions mentioned before ((5.13), (5.14), (5.24)), we have

$$
\begin{aligned}
\theta^{j}(t, x)= & -e^{-\beta x / \varepsilon} u^{j}(t, 0)+(-1)^{j+1}\left(\frac{\beta x}{\varepsilon}\right)^{j} e^{-\beta x / \varepsilon} \frac{\frac{\partial^{j}}{\partial t^{j}} u^{0}(t, 0)}{\beta^{2 j} j !} \\
& +\sum_{k=1}^{j-1} A_{j k}\left(\frac{\beta x}{\varepsilon}\right)^{k} e^{-\beta x / \varepsilon}+\text { e.s.t. }
\end{aligned}
$$

where the $A_{j k}$ are linear combinations of the $\frac{\partial^{j}}{\partial t^{j}} u^{0}(t, 0)$ and are independent of $\varepsilon$ and $x$.

Proof. We have already proved that (5.54) is true for $j=0$ and 1 . We now proceed using an induction argument.

Assuming that (5.54) is true for $j$, we prove that it is true for $j+1$. 
It is easy to see that the influence of an exponentially small term on $\theta^{j+1}$ is still exponentially small. Thus we have, using the stretched coordinate $X=\frac{\beta x}{\varepsilon}$ and (5.20), and the induction hypothesis:

$$
\begin{aligned}
-\frac{\partial^{2} \theta^{j+1}}{\partial X^{2}}-\frac{\partial \theta^{j+1}}{\partial X}= & -\frac{1}{\beta^{2}} \frac{\partial \theta^{j}}{\partial t} \\
= & \frac{1}{\beta^{2}} e^{-X} \frac{\partial u^{j}(t, 0)}{\partial t^{j}}+(-1)^{j+2} X^{j} e^{-X} \frac{\frac{\partial^{j+1}}{\partial t^{j+1}} u^{0}(t, 0)}{\beta^{2 j+2} j !} \\
& -\sum_{k=1}^{j-1} \frac{1}{\beta^{2}} \frac{\partial A_{j k}}{\partial t} X^{k} e^{-X}+\text { e.s.t. } \\
\left.\theta^{j+1}\right|_{X}= & \frac{\beta L}{\varepsilon}=0,\left.\quad \theta^{j+1}\right|_{X=0}=-\left.u^{j+1}\right|_{X=0} .
\end{aligned}
$$

It is then desirable to consider the problem

$$
-\frac{\partial^{2} \theta}{\partial X^{2}}-\frac{\partial \theta}{\partial X}=X^{k} e^{-X}
$$

Notice that

$$
\theta=\sum_{l=1}^{k+1} \frac{k !}{l !} X^{l} e^{-X}
$$

is a special solution to the previous equation. Hence we may define

$$
\begin{aligned}
\text { (5.59) } \theta_{1}^{j+1}= & \frac{1}{\beta^{2}} X e^{-X} \frac{\partial u^{j}(t, 0)}{\partial t}+(-1)^{j+2} \sum_{l=1}^{j+1} \frac{j !}{l !} X^{l} e^{-X} \frac{\frac{\partial^{j+1}}{\partial t^{j+1}} u^{0}(t, 0)}{\beta^{2 j+2} j !} \\
& -\sum_{k=1}^{j-1}\left(\sum_{l=1}^{k+1} \frac{k !}{l !} X^{l} e^{-X}\right) \frac{1}{\beta^{2}} \frac{\partial A_{j k}}{\partial t}, \\
= & \frac{1}{\beta^{2}} X e^{-X} \frac{\partial u^{j}(t, 0)}{\partial t}+(-1)^{j+2} X^{j+1} e^{-X} \frac{\frac{\partial^{j+1}}{\beta^{2 j+1}} u^{0}(t, 0)}{\beta^{2 j+2}(j+1) !} \\
& +(-1)^{j+2} \sum_{l=1}^{j} \frac{j !}{l !} X^{l} e^{-X} \frac{\frac{\partial^{j+1}}{\partial t^{j+1}} u^{0}(t, 0)}{\beta^{2 j+2} j !}-\sum_{k=1}^{j-1}\left(\sum_{l=1}^{k+1} \frac{k !}{l !} X^{l} e^{-X}\right) \frac{1}{\beta^{2}} \frac{\partial A_{j k}}{\partial t} \\
= & \frac{1}{\beta^{2}} X e^{-X} \frac{\partial u^{j}(t, 0)}{\partial t}+(-1)^{j+2} X^{j+1} e^{-X} \frac{\frac{\partial^{j+1}}{\partial t^{j+1}} u^{0}(t, 0)}{\beta^{2 j+2}(j+1) !}+\sum_{k=1}^{j} X^{k} e^{-X} A_{j+1, k} .
\end{aligned}
$$

Here

$$
A_{j+1, k}=(-1)^{j+2} \frac{1}{k !} \frac{\frac{\partial^{j+1}}{\partial t^{j+1}} u^{0}(t, 0)}{\beta^{2 j+2}}-\sum_{l=k-1}^{j-1} \frac{l !}{k !} \frac{1}{\beta^{2}} \frac{\partial A_{j l}}{\partial t} .
$$


Thus $\theta^{j+1}-\theta_{1}^{j+1}$ satisfies the equation

$$
\begin{aligned}
& -\frac{\partial^{2}\left(\theta^{j+1}-\theta_{1}^{j+1}\right)}{\partial X^{2}}-\frac{\partial\left(\theta^{j+1}-\theta_{1}^{j+1}\right)}{\partial X}=e . s . t . \\
& \left.\left(\theta^{j+1}-\theta_{1}^{j+1}\right)\right|_{X=\frac{\beta L}{\varepsilon}}=\text { e.s.t. },\left.\quad\left(\theta^{j+1}-\theta_{1}^{j+1}\right)\right|_{X=0}=-\left.u^{j+1}\right|_{X=0} .
\end{aligned}
$$

Consider now

$$
\tilde{\theta}^{j+1}=\theta_{1}^{j+1}+u^{j+1}(t, 0) e^{-\beta x / \varepsilon}
$$

We have

$$
\begin{aligned}
& -\frac{\partial^{2}\left(\theta^{j+1}-\tilde{\theta}^{j+1}\right)}{\partial X^{2}}-\frac{\partial\left(\theta^{j+1}-\tilde{\theta}^{j+1}\right)}{\partial X}=\text { e.s.t. } \\
& \left.\left(\theta^{j+1}-\tilde{\theta}^{j+1}\right)\right|_{X=\frac{\beta L}{\varepsilon}}=\text { e.s.t. },\left.\left(\theta^{j+1}-\tilde{\theta}^{j+1}\right)\right|_{X=0}=0 .
\end{aligned}
$$

This implies

$$
\theta^{j+1}=\tilde{\theta}^{j+1}+\text { e.s.t. }
$$

equivalently, thanks to (5.59), (5.60) and (5.63), we obtain (5.54). This completes the proof of the theorem.

We now turn to the validity in the interior of the naive approximation of $u^{\varepsilon}$ by its inviscid limit $u^{0}$.

THEOREM 7. Let $u^{\varepsilon}$ and $u^{0}$ be the solutions of the "viscous" and "inviscid" problems respectively. Then there exists a constant $\kappa$, independent of $\varepsilon$, such that

$$
\left\|u^{\varepsilon}-u^{0}\right\|_{L^{\infty}\left(0, T ; L^{2}(\Omega)\right)} \leq \kappa \varepsilon^{\frac{1}{2}} .
$$

Moreover, for $0<\delta<L$, there exists a constant $\kappa_{\delta}$, independent of $\varepsilon$, such that

$$
\left\|u^{\varepsilon}-u^{0}\right\|_{L^{\infty}((0, T) \times(\delta, L))} \leq \kappa_{\delta} \varepsilon^{\frac{1}{2}} .
$$

Proof. The proofs are a simple combination of our convergence results in Theorem 5 and our explicit form for the correctors given in Theorem 6 . Indeed,

$$
\begin{aligned}
\left\|u^{\varepsilon}-u^{0}\right\|_{L^{\infty}\left(0, T ; L^{2}(\Omega)\right)} \leq & \left\|u^{\varepsilon}-u^{0}-\theta^{0}\right\|_{L^{\infty}\left(0, T ; L^{2}(\Omega)\right)}+\left\|\theta^{0}\right\|_{L^{\infty}\left(0, T ; L^{2}(\Omega)\right)}, \\
& (\text { Thanks to }(5.33) \text { and }(5.54)) \\
\leq & \kappa \varepsilon+\kappa \varepsilon^{\frac{1}{2}} \\
\leq & \kappa \varepsilon^{\frac{1}{2}} .
\end{aligned}
$$

Likewise

$$
\begin{aligned}
\left\|u^{\varepsilon}-u^{0}\right\|_{L^{\infty}((0, T) \times(\delta, L))} \leq & \left\|u^{\varepsilon}-u^{0}-\theta^{0}\right\|_{L^{\infty}((0, T) \times(\delta, L))}+\left\|\theta^{0}\right\|_{L^{\infty}((0, T) \times(\delta, L))} \\
& (\text { Thanks to }(5.38) \text { and }(5.54)) \\
\leq & \kappa_{\delta} \varepsilon+\kappa_{\delta} \varepsilon^{\frac{1}{2}} \\
\leq & \kappa_{\delta} \varepsilon^{\frac{1}{2}}
\end{aligned}
$$

This ends the proof. $\square$ 
Acknowledgments. The work of Cheng and Temam was partially supported by the National Science Foundation under grant DMS-9705229 and the Research Fund of Indiana University. Cheng also acknowledges the support of an Eberhard Hopf Fellowship from Indiana University. The work of Wang was partially supported by the National Science Foundation under grant DMS-9971986 and a Faculty Development Fund from the College of Liberal Arts and Sciences of the Iowa State University. Cheng also acknowledges helpful comments from Randall Bramley and Michael Jolly.

\section{REFERENCES}

S. N. Alekseenko, 1994, Existence and asymptotic representation of weak solutions to the flowing problem under the condition of regular slippage on solid walls, Siberian Math. Journ., vol. 35, issue 2, pp. 209-229.

U. M. Ascher, R. M. M. MattheiJ, And R. D. Russell, 1988, Numerical Solution of Boundary Value Problems for Ordinary Differential Equations, Prentice Hall Inc., Englewood Cliffs, NJ.

G. K. Batchelor, 1967, An Introduction to Fluid Dynamics, Cambridge University Press, Cambridge.

W. F. Cheng, 1999, An Under-resolved Scheme for a Class of Partial Differential Equations with Boundary Layer, Ph.D Thesis, Indiana University, Bloomington.

W. F. Cheng And R. Temam, 2000, Numerical approximation of a class of elliptic problems with boundary layers, to appear.

Philippe G. Ciarlet, 1978, The Finite Element Method for Elliptic Problems, North-Holland Pub. Co., Amsterdam, New York.

Philippe G. Ciarlet, 1976, Numerical Analysis of the Finite Element Method, Les Presses de L'Université de Montréal, Quebec.

M. VAN DyKe, 1964, Perturbation Methods in Fluid Mechanics, Academic Press, New-York.

W. Eckhaus, 1979, Asymptotic Analysis of Singular Perturbations, North-Holland.

G. Fix And M. GunzBurger, 1977, Downstream boundary conditions for viscous flow problems, Comp. \& Maths. with Appls., vol. 3, pp. 53-63.

K. O. FRIEDRICHS, 1941, The mathematical structure of the boundary layer problem, in Fluid Dynamics, R. von Mises and K. O. Friedrichs, eds., Brown Univ., Providence, RI (reprinted by Springer-Verlag, New York, 1971), pp. 171-174.

M. Gisclon AND D. SERRE, 1994, Edute des conditions aux limites pour un systeme strictement hyperbolique, via l'approximation parabolique, C. R. Acad. Sci. Paris, Serie I, vol. 319, pp. $377-382$.

E. GRenIER AND O. GUES, 1998, Boundary layers for viscous perturbations of noncharacteristic quasilinear hyperbolic problem, J. Differential Equations, vol. 143, no. 1, pp. 110-146.

J. Hale, 1980, Ordinary Differential Equations Wiley, New York.

T. Y. Hou, X. WU, AND Z. CAI, 1999, Convergence of a multiscale finite element method for elliptic problems with rapidly oscillating coefficients, Math. Comp., vol. 68, no. 227, pp. 913-943.

F. John, 1982, Partial Differential Equations, 4th edition, Springer, New York.

O. A. Ladyzhenskaya, 1969, The Mathematical Theory of Viscous Incompressible Flows, 2nd ed, Gordon and Breach, New York.

P. Lagerström, 1988, Matched Asymptotics Expansion, Ideas and Techniques, Springer-Verlag, New York.

H. LAMB, 1945, Hydrodynamics, reprinted by Dover, New-York.

L. Landau And E. Lifschitz, 1954, Fluid Mechanics, Addison-Wesley.

J. L. Lions, 1973, Perturbations Singulières Dans les Problèmes aux Limites et en Contrôle Optimal, Lecture Notes in Math 323, Springer-Verlag, New-York.

H. K. Moffatt, 1977, Six lectures on general fluid dynamics and two on hydromagnetic dynamo theory, in Fluid Dynamics, Cours de l'Ecole d'Eté de Physique Théorique, Les Houches, R. Balian and J. L. Peube, ed., Gordon and Breach Science Publishers, New-York.

O. Oleinik, 1963, The Prandtl system of equations in boundary layer theory, Dokl. Akad. Nauk S.S.S.R., vol. 150 , pp. $28-31$.

O. A. Oleinik And V. N. Samokhin, 1999, Mathematical Models in Boundary Layer Theory, Chapman \& Hall, Boca Raton, FL. 
L. PRANDTL, 1905, Veber Flüssigkeiten bei sehr kleiner Reibung, in Verh. III Intern. Math Kongr. Heidelberg, Teuber, Leibzig, pp. 484-491.

R. Temam, 1982, Behaviour at time $t=0$ of the solutions of semi-linear evolution equations, J. Diff. Equ., vol. 17, pp. 73-92.

R. Temam, 1984, Navier-Stokes Equations: Theory and Numerical Analysis, North-Holland, Amsterdam.

R. Temam AND X. WANG, 1996, Asymptotic analysis of Oseen type equations in a channel at high Reynolds number, Indiana Univ. Math. J., vol. 45, no. 3, pp. 863-916.

R. Temam AND X. WANG, Boundary layers in channel flow with injection and suction, Applied Mathematics Letters, to appear.

R. TEMAM AND X. WANG, Boundary layers associated with incompressible Navier-Stokes equations: The noncharacteristic boundary case, to appear.

M. I. VISHIK AND L. A. LYUSTERNIK, 1957, Regular degeneration and boundary layer for linear differential equations with small parameter, Uspekki Mat. Nauk, vol. 12, pp. 3-122.

Z. XIN, 1998, Viscous boundary layers and their stability (I), J. Partial Diff. Equations., vol. 11, pp. $97-124$. 